\title{
OPEN Polar lipidomic profile shows Chlorococcum amblystomatis as a promising source of value-added lipids
}

\author{
Tiago A. Conde ${ }^{1}$, Daniela Couto ${ }^{1,2}$, Tânia Melo ${ }^{1,2}$, Margarida Costa ${ }^{3}$, Joana Silva ${ }^{3}$, \\ M. Rosário Domingues ${ }^{1,2}$ \& Pedro Domingues ${ }^{1 \triangle}$
}

There is a growing trend to explore microalgae as an alternative resource for the food, feed, pharmaceutical, cosmetic and fuel industry. Moreover, the polar lipidome of microalgae is interesting because of the reports of bioactive polar lipids which could foster new applications for microalgae. In this work, we identified for the first time the Chlorococcum amblystomatis lipidome using hydrophilic interaction liquid chromatography-high resolution electrospray ionization- tandem mass spectrometry (HILIC-HR-ESI-MS/MS). The Chlorococcum amblystomatis strain had a lipid content of $20.77 \%$ and the fatty acid profile, determined by gas chromatography-mass spectrometry, has shown that this microalga contains high amounts of omega-3 polyunsaturated fatty acids (PUFAs). The lipidome identified included 245 molecular ions and 350 lipid species comprising 15 different classes of glycolipids (6), phospholipids (7) and betaine lipids (2). Of these, 157 lipid species and the main lipid species of each class were esterified with omega-3 PUFAs. The lipid extract has shown antioxidant activity and anti-inflammatory potential. Lipid extracts also had low values of atherogenic $(0.54)$ and thrombogenic index ( 0.27$)$. In conclusion, the lipid extracts of Chlorococcum amblystomatis have been found to be a source of lipids rich in omega-3 PUFAs for of great value for the food, feed, cosmetic, nutraceutical and pharmaceutical industries.

Reducing the impact of climate change is one of the main challenges of this decade, which has increased the demand to move to more environmental and sustainable resources ${ }^{1,2}$. Microalgae represent a sustainable ${ }^{3,4}$ and versatile $^{5,6}$ resource with exploitation potential for different industries (for example food, feed, nutraceutical, cosmetic, pharmaceutical $)^{7}$. These unicellular organisms are found not only in aquatic systems but also in terrestrial systems, with a wide range of catalogued species ${ }^{8,9}$. Microalgae are valued for their photosynthetic efficiency, low water requirement, fast growth and high biomass production ${ }^{10}$, which allows efficient biomass production with lower resource consumption that meet industry's needs. Also, microalgae grow with greater photosynthetic efficiency than higher plants and do not require arable land to be cultivated ${ }^{11}$. Microalgae have been used for different applications, namely, as direct food, as food nutrients, for animal feed, as sources of bioactive products for the pharmaceutical industry, and as a source of lipids for the production of biodiesel ${ }^{5}$. For example, microalgae can consume nutrients that are in excess in the agri-food industry waste streams, such as nitrogen and phosphorous ${ }^{12}$.. In terms of nutrients, they are a rich source of essential amino acids, vitamins, minerals, pigments and lipids, namely polyunsaturated fatty acids (PUFAs) ${ }^{13}$. They are considered a promising source of bioactive components due to their antioxidant, anti-inflammatory, anti-obesity, anti-tumour, antiviral and anti-bacterial properties ${ }^{14}$. Among the bioactive compounds described, phospholipids (PL) seem to be interesting ingredients as the main vector of omega-3 fatty acids (FA) ${ }^{15}$ and glycolipids (GL) as promising bioactive phytochemicals ${ }^{16}$. There is a large body of evidence suggesting that omega-3 PUFAs esterified in polar lipids have important physiological properties. For example, PL and GL containing omega-3 FA, were associated with antioxidant and antiinflammatory properties, and with cardiovascular protection ${ }^{17}$ and also that PLs esterified in omega-3 PUFAs are excellent delivery vehicles ${ }^{18,19}$. In addition, GLs with PUFAs have shown anti-inflammatory potential with nitric oxide release inhibitory activity ${ }^{20,21}$ while DGDGs and SQDGs from algae showed chemotherapeutic potential ${ }^{22}$

${ }^{1}$ Department of Chemistry, Mass Spectrometry Centre, LAOV REQUIMTE, University of Aveiro, Santiago University Campus, 3810-193 Aveiro, Portugal. '2Department of Chemistry, CESAM-Centre for Environmental and Marine Studies, University of Aveiro, Santiago University Campus, 3810-193 Aveiro, Portugal. ${ }^{3}$ R\&D Department, Allmicroalgae Natural Products S.A., Rua 25 de Abril 19, 2445-287 Pataias, Portugal. ${ }^{\circledR}$ email: p.domingues@ua.pt 
and SQDG esterified in EPA also displays an anti-proliferative effect ${ }^{23}$. Very recent studies have focused on the identification of polar lipids from the most commercially used microalgae, such as Chlorella sp. and Chlorella vulgaris $^{24,25}$, Nannochloropsis oceanica and Nannochloropsis oculata ${ }^{26,27}$, and Phaeodactylum tricornutum ${ }^{28}$. However, polar lipids are scarcely studied compared to other ingredients derived from microalgae, an even larger number of microalgae lipidome remains unknown, unexplored or poorly studied, hampering the full biotechnological exploitation of this resource.

Chlorococcum sp. is a green microalga which belongs to the phylum Chlorophyta, class Chlorophyceae, order Chlorococcaceae and family Chlorococcaceae, and it can be cultivated under photoautotrophic and mixotrophic conditions ${ }^{29}$. The biotechnological potential of this microalga remains poorly explored ${ }^{29}$. Chlorococcum sp. has been described as a useful tool in carbon sequestration, lipid flocculation in wastewater ${ }^{30}$ and carotenoid production $^{31}$, a good source for the production of biodiesel ${ }^{32}$ and useful for the food industry ${ }^{33,34}$. The production of lutein from Chlorococcum citriforme has already been patented ${ }^{35}$. Regarding its biochemical composition, a total biomass dry weight content of proteins of $13.08-40 \%{ }^{32,36}$, carbohydrates of $3.42-40 \%^{32,36}$, chlorophylls of $0.4-1.5 \%{ }^{32}$, carotenoids of $3.2-12.6 \mathrm{mg} / \mathrm{g}^{37}$ and lipids of $8.71-32.3 \%{ }^{32,36,38-40}$ has been described. In addition to its high lipid content, the FA composition of the Chlorococcum strains studied to date, included good amounts of omega-3 fatty acids ${ }^{33}$ suggesting the possibility of added-value lipids that needs to be explored.

The interest of knowing the lipidome of green microalgae, either to optimize the production of biodiesel or to produce lipids with added value, has led to the complete profiling of several microalgae ${ }^{16,27}$. As such, the complete lipidome has been reported for certain green microalgae belonging to the phylum Chlorophyta, such as Haematococcus sp. ${ }^{27}$, Chlamydomonas sp. ${ }^{41}$, Scenedesmus sp. ${ }^{24}$, Chlorella $\mathrm{sp}^{42}$ and Chlorella kessleri ${ }^{43}$. Microalgal lipids have received much attention due to their high abundance of omega-3 and omega-6 PUFAs, and, more recently, due to their polar lipids content (e.g. monogalactosyldiacylglycerol [MGDG], digalactosyldiacylglycerol [DGDGs]), in which the PUFAs are esterified. These glycolipids are plastid lipids, therefore abundant in green microalgae. Also, these lipids were reported to have desired bioactive properties, such as antibacterial, antioxidant and anti-inflammatory activities ${ }^{16}$. PUFAs were already described in Chlorococcum sp. ${ }^{32,33,38,39}$, however, the polar lipidome of this microalga remains to be described, preventing its full exploitation. Thus, to fill this gap, in the present work, we have analysed and characterized in detail the polar lipidome of Chlorococcum amblystomatis, cultivated in autotrophic conditions, using a lipidomic approach based on the hydrophilic interaction liquid chromatography-electrospray ionization-high resolution mass spectrometry (HILIC-HR-ESI-MS). We also explored the bioactive potential of lipid extracts of Chlorococcum amblystomatis to inhibit COX-2 activity and its antioxidant properties, using commercial kits, to promote valorisation of this microalga as a source of bioactive lipids for the food, feed, cosmetics or pharmaceutical industries.

\section{Results}

Total lipid content and fatty acid composition of Chlorococcum amblystomatis. The lipid extracts of Chlorococcum amblystomatis corresponded to an average yield of $20.77 \pm 0.57 \%$ of microalgal biomass. The fatty acid composition of Chlorococcum amblystomatis identified by GC-MS showed that C16:0 was the most abundant FA (23.0 $\pm 0.8 \%)$, followed by C18:3 $n-3(19.4 \pm 0.6 \%), \mathrm{C} 18: 0$ (13.9 $\pm 2.3 \%), \mathrm{C} 16: 4 n-3$ $(11.1 \pm 0.6 \%), \mathrm{C} 20: 5 n-3(8.9 \pm 0.6 \%)$ and C16:1 $n-9(7.5 \pm 0.3 \%)$ (Table 1). The remaining identified FAs had relative abundances of less than 5\%. This microalga had a total omega- 3 fatty acid content of $43.2 \%$.

To assess the potential health benefits of Chlorococcum amblystomatis, the atherogenic (AI), thrombogenic (TI), hypocholesterolemic/hypercholesterolemic $(\mathrm{h} / \mathrm{H})$ and the PUFA $n-6 /$ PUFA $n-3$ indices were calculated. The AI and TI of the lipid extracts were 0.5 and 0.3 , respectively, while the $\mathrm{h} / \mathrm{H}$ ratio was 1.4 and the $n-6 / n-3$ ratio was 0.1 .

Polar lipid composition of Chlorococcum amblystomatis. The polar lipid profile was revealed by hydrophilic interaction liquid chromatography-high resolution electrospray mass spectrometry (HILIC-HRESI-MS and HILIC-HR-ESI-MS/MS) (Supplementary file S1). Using this approach, a total of 245 molecular ions, with a minimum of 350 molecular species, belonging to 15 classes of lipids were identified. Of these, 101 molecular ions were glycolipids (GL), 54 molecular ions were betaine lipids (BL), 89 molecular ions were phospholipids (PL), and 1 molecular ion was an inositol phosphoceramide (PI-Cer) (Tables 2 and 3). Figures explaining how the MS/MS data were interpreted can be found in the Supplementary Figures S1-S13. The $\mathrm{m} / z$ of the fragment ions used to identify each molecular ion and each molecular species can be found in Supplementary Table S1 and S2.

The PL classes identified included phosphatidylcholine (PC), lysophosphatidylcholine (LPC), phosphatidylethanolamine (PE) and lysophosphatidylethanolamine (LPE), identified in the HILIC-HR-ESI-MS analysis as the molecular ion $[\mathrm{M}+\mathrm{H}]^{+}$(Fig. 1); and phosphatidylglycerol (PG) and phosphatidylinositol (PI) identified as the negative molecular ion, $[\mathrm{M}-\mathrm{H}]^{-}$(Fig. 2). The only sphingolipid species found in Chlorococcum amblystomatis was a PI-Cer, which has been identified in the analysis on the negative ion mode. A total of 32 molecular ions of PC, 11 of LPC, 13 of PE, 9 of LPE, 22 of PG, 2 of PI and 1 of PI-Cer were recognized. Analysis of the MS/MS data allowed to recognize that each ion can correspond to several molecular species, with different combinations of fatty acyl chains, but with the same (C:N) (Supplementary Table S2). The molecular species identified per class were of 39 PC, 11 LPC, 14 PE, 9 LPE, 32 PG, 2 PI, and 1 PI-Cer.

As previously indicated, the identification of the lipid species was based on the mass accuracy, retention time and interpretation of MS/MS data. The interpretation of the MS/MS data of the molecular ions of the PC, LPC, $\mathrm{PE}$ and LPE species allowed to confirm their identity (Supplementary Table S2). This was done by confirming for each species the presence of the polar head groups, in particular by the presence of the product ion at $\mathrm{m} / \mathrm{z}$ 184 (for PC and LPC) and fragment ions arising from the neutral loss of $141 \mathrm{Da}$ (for PE and LPE) ${ }^{16,44-48}$. On the 


\begin{tabular}{|l|l|}
\hline Fatty acids & Relative abundance (\%) \pm SD \\
\hline C12:0 & $0.1 \pm 0.0$ \\
\hline C14:0 & $1.5 \pm 0.2$ \\
\hline C15:0-iso & $0.0 \pm 0.0$ \\
\hline C15:0 & $0.2 \pm 0.0$ \\
\hline C16:0 & $23.0 \pm 0.8$ \\
\hline C16:1 $\Delta^{9}$ & $7.5 \pm 0.3$ \\
\hline C16:1 $\Delta^{11}$ & $0.1 \pm 0.0$ \\
\hline C16:2 $\Delta^{7.10}(n-6)$ & $0.3 \pm 0.0$ \\
\hline C16:3 $\Delta^{4,7,10}(n-6)$ & $0.6 \pm 0.1$ \\
\hline C17:1 & $0.1 \pm 0.0$ \\
\hline C16:3 $\Delta^{7,10,13}(n-3)$ & $0.6 \pm 0.1$ \\
\hline C16:4 $\Delta^{4,7,10,13}(n-3)$ & $11.1 \pm 0.6$ \\
\hline C18:0 & $13.9 \pm 2.3$ \\
\hline C18:1 $\Delta^{9}$ & $1.6 \pm 0.1$ \\
\hline C18:1 $\Delta^{11}$ & $3.6 \pm 0.2$ \\
\hline C18:2 $\Delta^{9,12}(n-6)$ & $2.3 \pm 0.2$ \\
\hline C18:3 $\Delta^{6,9,12}(n-6)$ & $1.0 \pm 0.1$ \\
\hline C18:3 $\Delta^{9,12,15}(n-3)$ & $19.4 \pm 0.6$ \\
\hline C18:4 $\Delta^{6,9,12,15}(n-3)$ & $3.2 \pm 0.2$ \\
\hline C20:0 & $0.2 \pm 0.0$ \\
\hline C20:4 $\Delta^{5,8,11,14}(n-6)$ & $1.1 \pm 0.1$ \\
\hline C20:5 $\Delta^{5,8,11,14,17}(n-3)$ & $8.9 \pm 0.6$ \\
\hline$\Sigma$ SFA & 38.8 \\
\hline$\sum$ MUFAs & 12.9 \\
\hline$\sum$ PUFAs & 48.4 \\
\hline$\Sigma(n-3)$ & 43.2 \\
\hline$\Sigma(n-6)$ & 5.2 \\
\hline n-6/n-3 ratio & 0.1 \\
\hline AI & 0.5 \\
\hline TI & 0.3 \\
\hline (h/H) & 1.4 \\
\hline
\end{tabular}

Table 1. Fatty acid profile identified in the total lipid extract of Chlorococcum amblystomatis by GC-MS. Values are expressed in relative abundance (\%) and represent the mean of five analytical samples \pm standard deviation (SD). SFA saturated fatty acids, MUFAs monounsaturated fatty acids, PUFAs polyunsaturated fatty acids, $A I$ atherosclerotic index, $T I$ thrombogenic index, $(h / H)$ (hypocholesterolemic/hypercholesterolemic) ratio.

other hand, the complementary analysis of the molecular ions on the HILIC-HR-ESI-MS/MS spectra acquired in negative ion mode, observed as $\left[\mathrm{M}+\mathrm{CH}_{3} \mathrm{COO}\right]^{-}$ions for PC and $\mathrm{LPC}$ and $[\mathrm{M}-\mathrm{H}]^{-}$ions for PE and LPE, allowed to assign the fatty acyl composition. This was done by observing the presence of the $\mathrm{RCOO}^{-}$carboxylate fragment anions of the fatty acids esterified in the glycerol backbone ${ }^{16,44-48}$.

The HILIC-HR-ESI-MS/MS spectra of the negative molecular ions belonging to the PG, PI and PI-Cer classes allowed to confirm the presence of the corresponding polar heads by observing for each species the product ions at $m / z 171$ (for PG) and $m / z 241$ (for PI and PI-Cer), and identify the fatty acyl chains by the presence of $\mathrm{RCOO}^{-}$carboxylate anions (for PG and PI) ${ }^{16,44-48}$ (Supplementary Table S2). The only PI-Cer detected was identified by the accurate mass measurement, the retention time, and the presence of the polar head at $m / z 241$. The most abundant species in each phospholipid class were: PC(34:2) identified as PC(16:1-18:1) and PC(16:0-18:2); LPC(16:1); PE(34:4) identified as PE(16:0-18:4); LPE(16:0); PG(34:3) identified as PG(16:0-18:3); and $\mathrm{PI}(32: 1)$ identified as $\mathrm{PI}(16: 0-16: 1)$.

Among the PG molecular ions identified, a few oxidized PG species were found (Table 3), the most abundant being PG(34:2-OH), identified as PG(18:0-OH-16:2). All the oxidized PG species were identified based on exact mass measurements, retention time and MS/MS spectra analysis (Supplementary Table S3). In the MS/MS spectra, the presence of mass shifts of +16 Da relative to the $[\mathrm{M}-\mathrm{H}]^{-}$ions and of $\mathrm{RCOO}^{-}$carboxylate hydroxyl anions was indicative of the formation of hydroxy derivatives ${ }^{49,50}$. No product ions with oxidized polar head groups were observed ${ }^{45}$.

The betaine lipids identified in the HILIC-HR-ESI-MS data included the classes of monoacylglycerol-trimethylhomoserine (MGTS) and diacylglycerol-trimethylhomoserine (DGTS), and were identified in positive ion mode as $[\mathrm{M}+\mathrm{H}]^{+}$ions (Table 4, Fig. 3). A total of 38 molecular ions of DGTS and 16 molecular ions of MGTS were identified, corresponding to 68 molecular species of DGTS and 16 molecular species of MGTS. In 


\begin{tabular}{|l|l|l|l|}
\hline \multirow{2}{*}{ Lipid classes } & \multicolumn{4}{|l|}{ Chlorococcum amblystomatis } & \multicolumn{2}{l|}{} \\
\cline { 2 - 4 } & Molecular ions number & Lipid species number & Major species \\
\hline Glycolipids & 101 & 158 & - \\
\hline MGDG & 30 & 47 & MGDG(34:7) \\
\hline MGMG & 11 & 11 & MGMG(16:4) \\
\hline DGDG & 27 & 48 & DGDG(34:3) \\
\hline DGMG & 11 & 11 & DGMG(18:3) \\
\hline SQDG & 21 & 40 & SQDG(32:1) \\
\hline SQMG & 1 & 1 & SQMG(16:0) \\
\hline Phospholipids & 89 & 107 & - \\
\hline LPC & 11 & 11 & LPC(16:1) \\
\hline PC & 32 & 39 & PC(34:2) \\
\hline LPE & 9 & 9 & LPE(16:0) \\
\hline PE & 13 & 14 & PE(34:4) \\
\hline PG & 22 & 32 & PG(34:3) \\
\hline PI & 2 & 2 & PI(32:1) \\
\hline Betaine lipids & 54 & 84 & DGTS(34:4) \\
\hline DGTS & 38 & 68 & MGTS(18:4) \\
\hline MGTS & 16 & 16 & \\
\hline Sphingolipids & 1 & 1 & PI-Cer(d18:1/14:0) \\
\hline PI-Cer & 1 & 1 & - \\
\hline Total & 245 & 350 & \\
\hline
\end{tabular}

Table 2. Polar lipid classes identified in the total lipid extract of Chlorococcum amblystomatis by HILIC-HRESI-MS and MS/MS. The total number of molecular ions, lipid species identified in each class and lipid species per class is shown. $M G D G$ monogalactosyldiacylglycerol, $M G M G$ monogalactosylmonoacylglycerol, $D G D G$ digalactosyldiacylglycerol, $D G M G$ digalactosylmonoacylglycerol, $S Q D G$ sulfoquinovosyl diacylglycerol, $S Q M G$ sulfoquinovosyl monoacylglycerol, $L P C$ lysophosphatidylcholine, $P C$ phosphatidylcholine, $L P E$ lysophosphatidylethanolamine, $P E$ phosphatidylethanolamine, $P G$ phosphatidylglycerol, PI phosphatidylinositol, DGTS diacylglycerol-trimethylhomoserine, MGTS monoacylglyceroltrimethylhomoserine, $P I$-Cer inositol phosphoceramide.

the HILIC-HR-ESI-MS/MS spectra of MGTS and DGTS, as $[\mathrm{M}+\mathrm{H}]^{+}$ions, the presence of the polar head group in each species was confirmed by observation of the product ion at $m / z 236^{44,47}$, and the fatty acyl composition was determined by observation of the fragment ions resulting from the neutral losses of the fatty acyl chains as an acid $(-\mathrm{RCOOH})$ and ketene $(-\mathrm{R}=\mathrm{C}=\mathrm{O})$ derivatives ${ }^{44,47}$ (Supplementary Table $\left.\mathrm{S} 2\right)$.

The most abundant ions in each class of betaine lipids were MGTS (18:4) and DGTS (34:4) assigned as DGTS (18:4-16:0) and DGTS (18:3-16:1) (Table 4). Interestingly, among the identified DGTS molecular ions, oxidized DGTS molecular ions were also found, the most abundant being DGTS (34:7-OH), identified as a combination of two molecular species of DGTS (16:4-OH-18:3) and DGTS (16:3-OH-18:4). These oxidized ions were identified based on exact mass measurements, retention times, and by analysis of MS/MS spectra (Supplementary Table S3). The MS/MS of the oxidized DGTS molecular ions showed a neutral loss of $\mathrm{H}_{2} \mathrm{O}$ and the product ions formed due to the loss of oxidized fatty acyl chains (acid and keto derivatives). Also, the product ion characteristic of betaine lipids at $\mathrm{m} / z 236$ was present in all MS/MS spectra, therefore no product ions with oxidized polar head groups were observed.

The glycolipids identified in the Chlorococcum amblystomatis samples included monogalactosyldiacylglycerol (MGDG), monogalactosylmonoacylglycerol (MGMG), digalactosyldiacylglycerol (DGDG) and digalactosylmonoacylglycerol (DGMG), identified as $\left[\mathrm{M}+\mathrm{NH}_{4}\right]^{+}$ions (Fig. 4, Table 5). We also identified sulfoquinovosyl diacylglycerol lipids (SQDG) and a sulfoquinovosyl monoacylglycerol lipid (SQMG), identified as $[\mathrm{M}-\mathrm{H}]^{-}$ions (Fig. 5, Table 5). Analysis of the HILIC-HR-ESI-MS and MS/MS spectra identified the following number of molecular ions: 30 MGDG, 11 MGMG, 27 DGDG, 11 DGMG, 21 SQDG and 1 SQMG (Supplementary Table S2). From these molecular ions, we identified the following number of molecular species using MS/MS data: 47 MGDG, 11 MGMG, 48 DGDG, 11 DGMG, 40 SQDG and 1 SQMG.

The typical fragmentation observed in the HILIC-HR-ESI-MS/MS spectra of neutral glycolipids, as $\left[\mathrm{M}+\mathrm{NH}_{4}\right]^{+}$ions, allowed to confirm the polar head group by the neutral loss of $197 \mathrm{Da}$ (for MGDG) or $359 \mathrm{Da}$ (for DGDG), which corresponds to the loss of the carbohydrate moiety combined with the loss of $\mathrm{NH}_{3}{ }^{44,47}$. The assignment of the fatty acyl composition was corroborated by the presence of product ions corresponding to each fatty acyl group as acylium plus $74[\mathrm{RCO}+74]^{+}$ion $^{44,47}$ (Supplementary Table S2). On the other hand, the HILIC-HR-ESI-MS/MS spectra of the $[\mathrm{M}-\mathrm{H}]^{-}$ions of the acidic glycolipids (SQDG and SQMG), when available, showed the product ion of the sulfoquinovosyl polar head group at $\mathrm{m} / z 225$ and the fatty acyl composition was confirmed by the neutral loss of fatty acyl chains as carboxylic acid (-RCOOH) and by the presence of carboxylate $\mathrm{RCOO}^{-}$anions (Supplementary Table S2). 


\begin{tabular}{|c|c|c|c|c|c|}
\hline Lipid species (C:N) & Calculated $m / z$ & Observed $m / z$ & Error $(\mathrm{ppm})$ & Fatty acyl chains (C:N) & Formula \\
\hline \multicolumn{6}{|c|}{ LPC identified as $[\mathrm{M}+\mathrm{H}]^{+}$} \\
\hline LPC14:0) & 468.3090 & 468.3086 & -0.9 & $* *$ & $\mathrm{C}_{22} \mathrm{H}_{47} \mathrm{NO}_{7} \mathrm{P}$ \\
\hline $\operatorname{LPC}(16: 0)$ & 496.3403 & 496.3404 & 0.2 & $* *$ & $\mathrm{C}_{24} \mathrm{H}_{51} \mathrm{NO}_{7} \mathrm{P}$ \\
\hline $\operatorname{LPC}(16: 1)$ & 494.3247 & 494.3244 & -0.5 & $16: 1$ & $\mathrm{C}_{24} \mathrm{H}_{49} \mathrm{NO}_{7} \mathrm{P}$ \\
\hline $\operatorname{LPC}(16: 2)$ & 492.3090 & 492.3083 & -1.5 & $* *$ & $\mathrm{C}_{24} \mathrm{H}_{47} \mathrm{NO}_{7} \mathrm{P}$ \\
\hline LPC(18:1) & 522.3560 & 522.3561 & 0.3 & 18:1 & $\mathrm{C}_{26} \mathrm{H}_{53} \mathrm{NO}_{7} \mathrm{P}$ \\
\hline LPC(18:2) & 520.3403 & 520.3402 & -0.2 & 18:2 & $\mathrm{C}_{26} \mathrm{H}_{51} \mathrm{NO}_{7} \mathrm{P}$ \\
\hline $\operatorname{LPC}(18: 3)$ & 518.3247 & 518.3233 & -2.6 & * & $\mathrm{C}_{26} \mathrm{H}_{49} \mathrm{NO}_{7} \mathrm{P}$ \\
\hline LPC(18:4) & 516.3090 & 516.3067 & -4.5 & * & $\mathrm{C}_{26} \mathrm{H}_{47} \mathrm{NO}_{7} \mathrm{P}$ \\
\hline $\operatorname{LPC}(20: 3)$ & 546.3560 & 546.3560 & 0.1 & $* *$ & $\mathrm{C}_{28} \mathrm{H}_{53} \mathrm{NO}_{7} \mathrm{P}$ \\
\hline $\operatorname{LPC}(20: 4)$ & 544.3403 & 544.3398 & -0.9 & $* *$ & $\mathrm{C}_{28} \mathrm{H}_{51} \mathrm{NO}_{7} \mathrm{P}$ \\
\hline $\mathrm{LPC}(20: 5)$ & 542.3247 & 542.3241 & -1.0 & ${ }^{*}$ & $\mathrm{C}_{28} \mathrm{H}_{49} \mathrm{NO}_{7} \mathrm{P}$ \\
\hline \multicolumn{6}{|c|}{ PC identified as $[\mathrm{M}+\mathrm{H}]^{+}$} \\
\hline $\mathrm{PC}(28: 1)$ & 676.4917 & 676.4905 & -1.8 & * & $\mathrm{C}_{36} \mathrm{H}_{71} \mathrm{NO}_{8} \mathrm{P}$ \\
\hline $\mathrm{PC}(30: 0)$ & 706.5387 & 706.5371 & -2.2 & $* *$ & $\mathrm{C}_{38} \mathrm{H}_{77} \mathrm{NO}_{8} \mathrm{P}$ \\
\hline $\mathrm{PC}(30: 1)$ & 704.5230 & 704.5250 & 2.8 & $16: 1-14: 0$ & $\mathrm{C}_{38} \mathrm{H}_{75} \mathrm{NO}_{8} \mathrm{P}$ \\
\hline $\mathrm{PC}(30: 3)$ & 700.4917 & 700.4895 & -3.2 & * & $\mathrm{C}_{38} \mathrm{H}_{71} \mathrm{NO}_{8} \mathrm{P}$ \\
\hline $\mathrm{PC}(32: 1)$ & 732.5543 & 732.5539 & -0.6 & 16:1-16:0 & $\mathrm{C}_{40} \mathrm{H}_{79} \mathrm{NO}_{8} \mathrm{P}$ \\
\hline $\mathrm{PC}(32: 2)$ & 730.5387 & 730.5384 & -0.4 & 16:1-16:1 & $\mathrm{C}_{40} \mathrm{H}_{77} \mathrm{NO}_{8} \mathrm{P}$ \\
\hline $\mathrm{PC}(32: 3)$ & 728.5230 & 728.5230 & 0.0 & $16: 1-16: 2$ & $\mathrm{C}_{40} \mathrm{H}_{75} \mathrm{NO}_{8} \mathrm{P}$ \\
\hline $\mathrm{PC}(32: 4)$ & 726.5074 & \begin{tabular}{|l|l|}
726.5058 \\
\end{tabular} & -2.2 & * & $\mathrm{C}_{40} \mathrm{H}_{73} \mathrm{NO}_{8} \mathrm{P}$ \\
\hline $\mathrm{PC}(32: 5)$ & 724.4917 & 724.4908 & -1.3 & ** & $\mathrm{C}_{40} \mathrm{H}_{71} \mathrm{NO}_{8} \mathrm{P}$ \\
\hline $\mathrm{PC}(34: 1)$ & 760.5856 & 760.5842 & -1.9 & $16: 0-18: 1$ and $16: 1-18: 0$ & $\mathrm{C}_{42} \mathrm{H}_{83} \mathrm{NO}_{8} \mathrm{P}$ \\
\hline $\mathrm{PC}(34: 2)$ & 758.5700 & 758.5696 & -0.5 & $16: 1-18: 1$ and $16: 0-18: 2$ & $\mathrm{C}_{42} \mathrm{H}_{81} \mathrm{NO}_{8} \mathrm{P}$ \\
\hline $\mathrm{PC}(34: 3)$ & 756.5543 & 756.5542 & -0.2 & $16: 1-18: 2$ & $\mathrm{C}_{42} \mathrm{H}_{79} \mathrm{NO}_{8} \mathrm{P}$ \\
\hline $\mathrm{PC}(34: 4)$ & 754.5387 & 754.5371 & -2.1 & $16: 1-18: 3$ and $16: 2-18: 2$ & $\mathrm{C}_{42} \mathrm{H}_{77} \mathrm{NO}_{8} \mathrm{P}$ \\
\hline $\mathrm{PC}(34: 5)$ & 752.5230 & 752.5210 & -2.7 & * & $\mathrm{C}_{42} \mathrm{H}_{75} \mathrm{NO}_{8} \mathrm{P}$ \\
\hline $\mathrm{PC}(34: 6)$ & 750.5074 & 750.5064 & -1.3 & * & $\mathrm{C}_{42} \mathrm{H}_{73} \mathrm{NO}_{8} \mathrm{P}$ \\
\hline $\mathrm{PC}(34: 7)$ & 748.4917 & 748.4899 & -2.4 & $* *$ & $\mathrm{C}_{42} \mathrm{H}_{71} \mathrm{NO}_{8} \mathrm{P}$ \\
\hline $\mathrm{PC}(36: 2)$ & 786.6013 & 786.6004 & -1.1 & $* *$ & $\mathrm{C}_{44} \mathrm{H}_{85} \mathrm{NO}_{8} \mathrm{P}$ \\
\hline $\mathrm{PC}(36: 3)$ & 784.5856 & 784.5850 & -0.8 & $18: 1-18: 2$ & $\mathrm{C}_{44} \mathrm{H}_{83} \mathrm{NO}_{8} \mathrm{P}$ \\
\hline $\mathrm{PC}(36: 4)$ & 782.5700 & 782.5682 & -2.3 & $18: 2-18: 2$ and $16: 0-20: 4$ & $\mathrm{C}_{44} \mathrm{H}_{81} \mathrm{NO}_{8} \mathrm{P}$ \\
\hline $\mathrm{PC}(36: 5)$ & 780.5543 & 780.5528 & -2.0 & $18: 3-18: 2,16: 0-20: 5$ and $16: 1-20: 4$ & $\mathrm{C}_{44} \mathrm{H}_{79} \mathrm{NO}_{8} \mathrm{P}$ \\
\hline $\mathrm{PC}(36: 6)$ & 778.5387 & 778.5379 & -1.0 & $16: 1-20: 5$ & $\mathrm{C}_{44} \mathrm{H}_{77} \mathrm{NO}_{8} \mathrm{P}$ \\
\hline $\mathrm{PC}(36: 7)$ & 776.5230 & 776.5212 & -2.4 & * & $\mathrm{C}_{44} \mathrm{H}_{75} \mathrm{NO}_{8} \mathrm{P}$ \\
\hline $\mathrm{PC}(38: 5)$ & 808.5856 & 808.5843 & -1.6 & $18: 1-20: 4$ & $\mathrm{C}_{46} \mathrm{H}_{83} \mathrm{NO}_{8} \mathrm{P}$ \\
\hline $\mathrm{PC}(38: 6)$ & 806.5700 & 806.5690 & -1.2 & $18: 2-20: 4$ and $18: 1-20: 5$ & $\mathrm{C}_{46} \mathrm{H}_{81} \mathrm{NO}_{8} \mathrm{P}$ \\
\hline $\mathrm{PC}(38: 7)$ & 804.5543 & 804.5525 & -2.3 & $18: 2-20: 5$ & $\mathrm{C}_{46} \mathrm{H}_{79} \mathrm{NO}_{8} \mathrm{P}$ \\
\hline $\mathrm{PC}(38: 8)$ & 802.5387 & 802.5351 & -4.5 & ** & $\mathrm{C}_{46} \mathrm{H}_{77} \mathrm{NO}_{8} \mathrm{P}$ \\
\hline $\mathrm{PC}(38: 9)$ & 800.5230 & 800.5204 & -3.3 & $*$ & $\mathrm{C}_{46} \mathrm{H}_{75} \mathrm{NO}_{8} \mathrm{P}$ \\
\hline $\mathrm{PC}(40: 10)$ & 826.5387 & 826.5369 & -2.2 & * & $\mathrm{C}_{48} \mathrm{H}_{77} \mathrm{NO}_{8} \mathrm{P}$ \\
\hline $\mathrm{PC}(40: 5)$ & 836.6169 & 836.6180 & 1.3 & * & $\mathrm{C}_{48} \mathrm{H}_{87} \mathrm{NO}_{8} \mathrm{P}$ \\
\hline $\mathrm{PC}(40: 7)$ & 832.5856 & 832.5831 & -3.0 & $* *$ & $\mathrm{C}_{48} \mathrm{H}_{83} \mathrm{NO}_{8} \mathrm{P}$ \\
\hline $\mathrm{PC}(40: 8)$ & 830.5700 & 830.5674 & -3.1 & ${ }^{*}$ & $\mathrm{C}_{48} \mathrm{H}_{81} \mathrm{NO}_{8} \mathrm{P}$ \\
\hline $\mathrm{PC}(40: 9)$ & 828.5543 & 828.5520 & -2.8 & * & $\mathrm{C}_{48} \mathrm{H}_{79} \mathrm{NO}_{8} \mathrm{P}$ \\
\hline \multicolumn{6}{|c|}{ LPE identified as $[\mathrm{M}+\mathrm{H}]^{+}$} \\
\hline $\operatorname{LPE}(14: 0)$ & 426.2621 & 426.2615 & -1.3 & ${ }^{* *}$ & $\mathrm{C}_{19} \mathrm{H}_{41} \mathrm{NO}_{7} \mathrm{P}$ \\
\hline $\operatorname{LPE}(16: 0)$ & 454.2934 & 454.2928 & -1.2 & $16: 0$ & $\mathrm{C}_{21} \mathrm{H}_{45} \mathrm{NO}_{7} \mathrm{P}$ \\
\hline LPE(16:1) & 452.2777 & 452.2774 & -0.7 & * & $\mathrm{C}_{21} \mathrm{H}_{43} \mathrm{NO}_{7} \mathrm{P}$ \\
\hline LPE(16:4) & 446.2308 & 446.2303 & $-1 \mathrm{t} .0$ & $* *$ & $\mathrm{C}_{21} \mathrm{H}_{37} \mathrm{NO}_{7} \mathrm{P}$ \\
\hline LPE(18:1) & 480.3090 & 480.3092 & 0.4 & 18:1 & $\mathrm{C}_{23} \mathrm{H}_{47} \mathrm{NO}_{7} \mathrm{P}$ \\
\hline LPE(18:2) & \begin{tabular}{|l|}
478.2934 \\
\end{tabular} & \begin{tabular}{|l|l|}
478.2926 \\
\end{tabular} & -1.6 & $* *$ & $\mathrm{C}_{23} \mathrm{H}_{45} \mathrm{NO}_{7} \mathrm{P}$ \\
\hline LPE(18:3) & 476.2777 & 476.2774 & -0.7 & $*$ & $\mathrm{C}_{23} \mathrm{H}_{43} \mathrm{NO}_{7} \mathrm{P}$ \\
\hline LPE(18:4) & 474.2621 & 474.2618 & -0.6 & $18: 4$ & $\mathrm{C}_{23} \mathrm{H}_{41} \mathrm{NO}_{7} \mathrm{P}$ \\
\hline LPE(20:5) & 500.2777 & 500.2781 & 0.8 & $* *$ & $\mathrm{C}_{25} \mathrm{H}_{43} \mathrm{NO}_{7} \mathrm{P}$ \\
\hline \multicolumn{6}{|c|}{ PE identified as $[\mathrm{M}+\mathrm{H}]^{+}$} \\
\hline
\end{tabular}




\begin{tabular}{|c|c|c|c|c|c|}
\hline Lipid species (C:N) & Calculated $m / z$ & Observed $m / z$ & Error (ppm) & Fatty acyl chains (C:N) & Formula \\
\hline $\mathrm{PE}(30: 1)$ & 662.4761 & 662.4754 & -1.0 & $14: 0-16: 1$ & $\mathrm{C}_{35} \mathrm{H}_{69} \mathrm{NO}_{8} \mathrm{P}$ \\
\hline $\mathrm{PE}(30: 3)$ & \begin{tabular}{|l|}
658.4448 \\
\end{tabular} & 658.4422 & -3.9 & * & $\mathrm{C}_{35} \mathrm{H}_{65} \mathrm{NO}_{8} \mathrm{P}$ \\
\hline $\mathrm{PE}(32: 1)$ & 690.5074 & 690.5058 & -2.3 & $*$ & $\mathrm{C}_{37} \mathrm{H}_{73} \mathrm{NO}_{8} \mathrm{P}$ \\
\hline $\mathrm{PE}(32: 2)$ & \begin{tabular}{|l|l|}
688.4917 \\
\end{tabular} & \begin{tabular}{|l|l|}
688.4925 \\
\end{tabular} & 1.1 & $16: 1-16: 1$ & $\mathrm{C}_{37} \mathrm{H}_{71} \mathrm{O}_{8} \mathrm{NP}$ \\
\hline $\mathrm{PE}(32: 4)$ & 684.4604 & 684.4593 & -1.7 & 16:4-16:0 & $\mathrm{C}_{37} \mathrm{H}_{67} \mathrm{NO}_{8} \mathrm{P}$ \\
\hline $\mathrm{PE}(34: 1)$ & \begin{tabular}{|l|l|}
718.5387 \\
\end{tabular} & \begin{tabular}{|l|l|}
718.5353 \\
\end{tabular} & -4.7 & $16: 0-18: 1$ & $\mathrm{C}_{39} \mathrm{H}_{77} \mathrm{NO}_{8} \mathrm{P}$ \\
\hline $\mathrm{PE}(34: 2)$ & 716.5230 & 716.5219 & -1.6 & $16: 1-18: 1$ and $16: 0-18: 2$ & $\mathrm{C}_{39} \mathrm{H}_{75} \mathrm{NO}_{8} \mathrm{P}$ \\
\hline $\mathrm{PE}(34: 3)$ & 714.5074 & 714.5044 & -4.2 & * & $\mathrm{C}_{39} \mathrm{H}_{73} \mathrm{O}_{8} \mathrm{NP}$ \\
\hline $\mathrm{PE}(34: 4)$ & 712.4917 & 712.4901 & -2.3 & $16: 0-18: 4$ & $\mathrm{C}_{39} \mathrm{H}_{71} \mathrm{NO}_{8} \mathrm{P}$ \\
\hline $\mathrm{PE}(34: 5)$ & 710.4761 & 710.4754 & -1.0 & 16:1-18:4 & $\mathrm{C}_{39} \mathrm{H}_{69} \mathrm{NO}_{8} \mathrm{P}$ \\
\hline $\mathrm{PE}(36: 2)$ & 744.5543 & \begin{tabular}{|l|l|}
744.5535 \\
\end{tabular} & -1.1 & 18:1-18:1 & $\mathrm{C}_{41} \mathrm{H}_{79} \mathrm{O}_{8} \mathrm{NP}$ \\
\hline $\mathrm{PE}(36: 5)$ & 738.5074 & 738.5075 & 0.2 & $18: 4-18: 1$ & $\mathrm{C}_{41} \mathrm{H}_{73} \mathrm{O}_{8} \mathrm{NP}$ \\
\hline $\mathrm{PE}(36: 6)$ & \begin{tabular}{|l|}
736.4917 \\
\end{tabular} & \begin{tabular}{|l|}
736.4917 \\
\end{tabular} & 0.0 & * & $\mathrm{C}_{41} \mathrm{H}_{71} \mathrm{NO}_{8} \mathrm{P}$ \\
\hline \multicolumn{6}{|c|}{ PG identified as $[\mathrm{M}-\mathrm{H}]^{-}$} \\
\hline PG(30:0) & 693.4707 & 693.4700 & -1.0 & 14:0-16:0 & $\mathrm{C}_{36} \mathrm{H}_{70} \mathrm{O}_{10} \mathrm{P}$ \\
\hline PG(30:1) & 691.4550 & 691.4545 & -0.7 & ** & $\mathrm{C}_{36} \mathrm{H}_{68} \mathrm{O}_{10} \mathrm{P}$ \\
\hline PG(32:0) & 721.5020 & 721.5026 & 0.8 & ** & $\mathrm{C}_{38} \mathrm{H}_{74} \mathrm{O}_{10} \mathrm{P}$ \\
\hline PG(32:1) & 719.4863 & 719.4866 & 0.4 & $16: 1-16: 0$ and $14: 0-18: 1$ & $\mathrm{C}_{38} \mathrm{H}_{72} \mathrm{O}_{10} \mathrm{P}$ \\
\hline PG(32:2) & 717.4707 & 717.4703 & -0.6 & $16: 1-16: 1$ & $\mathrm{C}_{38} \mathrm{H}_{70} \mathrm{O}_{10} \mathrm{P}$ \\
\hline PG(34:1) & 747.5176 & 747.5181 & 0.7 & $16: 0-18: 1$ & $\mathrm{C}_{40} \mathrm{H}_{76} \mathrm{O}_{10} \mathrm{P}$ \\
\hline PG(34:2) & 745.5020 & 745.5011 & -1.2 & ** & $\mathrm{C}_{40} \mathrm{H}_{74} \mathrm{O}_{10} \mathrm{P}$ \\
\hline PG(34:3) & 743.4867 & 743.4868 & 0.1 & $16: 0-18: 3$ & $\mathrm{C}_{40} \mathrm{H}_{72} \mathrm{O}_{10} \mathrm{P}$ \\
\hline PG(34:4) & 741.4707 & 741.4711 & 0.5 & $16: 0-18: 4$ and $16: 1-18: 3$ & $\mathrm{C}_{40} \mathrm{H}_{70} \mathrm{O}_{10} \mathrm{P}$ \\
\hline PG(34:5) & 739.4550 & 739.4534 & -2.2 & $14: 0-20: 5$ and $16: 2-18: 3$ & $\mathrm{C}_{40} \mathrm{H}_{68} \mathrm{O}_{10} \mathrm{P}$ \\
\hline PG(36:2) & 773.5333 & 773.5337 & 0.5 & $18: 1-18: 1$ & $\mathrm{C}_{42} \mathrm{H}_{78} \mathrm{O}_{10} \mathrm{P}$ \\
\hline PG(36:5) & 767.4864 & 767.4863 & -0.1 & $16: 0-20: 5$ & $\mathrm{C}_{42} \mathrm{H}_{72} \mathrm{O}_{10} \mathrm{P}$ \\
\hline PG(36:6) & 765.4707 & 765.4715 & 1.0 & $16: 1-20: 5$ & $\mathrm{C}_{42} \mathrm{H}_{70} \mathrm{O}_{10} \mathrm{P}$ \\
\hline PG(38:5) & 795.5176 & 795.5197 & 2.6 & *** & $\mathrm{C}_{44} \mathrm{H}_{76} \mathrm{O}_{10} \mathrm{P}$ \\
\hline PG(32:2-OH) & 733.4656 & 733.4653 & -0.4 & 16:0-OH-16:2 & $\mathrm{C}_{38} \mathrm{H}_{70} \mathrm{O}_{11} \mathrm{P}$ \\
\hline PG(34:1-OH) & 763.5125 & 763.5115 & -1.3 & 18:0-OH-16:1 & $\mathrm{C}_{40} \mathrm{H}_{76} \mathrm{O}_{11} \mathrm{P}$ \\
\hline PG(34:2-OH) & 761.4969 & 761.4971 & 0.2 & $18: 0-\mathrm{OH}-16: 2$ & $\mathrm{C}_{40} \mathrm{H}_{74} \mathrm{O}_{11} \mathrm{P}$ \\
\hline PG(34:3-OH) & 759.4812 & 759.4808 & -0.5 & $\begin{array}{l}\text { 18:3-OH-16:0, 18:2-OH-16:1, 18:1-OH-16:2 } \\
\text { and 18:0-OH-16:3 }\end{array}$ & $\mathrm{C}_{40} \mathrm{H}_{72} \mathrm{O}_{11} \mathrm{P}$ \\
\hline PG(34:4-OH) & 757.4656 & 757.4653 & -0.4 & $\begin{array}{l}\text { 18:4-OH-16:0, 18:3-OH-16:1 and 18:2-OH- } \\
16: 2\end{array}$ & $\mathrm{C}_{40} \mathrm{H}_{70} \mathrm{O}_{11} \mathrm{P}$ \\
\hline PG(34:5-OH) & 755.4499 & 755.4480 & -2.5 & 18:3-OH-16:2 and 18:4-OH-16:1 & $\mathrm{C}_{40} \mathrm{H}_{68} \mathrm{O}_{11} \mathrm{P}$ \\
\hline PG(36:5-OH) & 783.4812 & 783.4828 & 2.0 & 16:0-OH-20:5 and 20:5-OH-16:0 & $\mathrm{C}_{42} \mathrm{H}_{72} \mathrm{O}_{11} \mathrm{P}$ \\
\hline $\mathrm{PG}(36: 6-\mathrm{OH})$ & 781.4656 & 781.4665 & 1.1 & $20: 5-\mathrm{OH}-16: 1$ & $\mathrm{C}_{42} \mathrm{H}_{70} \mathrm{O}_{11} \mathrm{P}$ \\
\hline \multicolumn{6}{|c|}{ PI identified as $[\mathrm{M}-\mathrm{H}]^{-}$} \\
\hline $\operatorname{PI}(32: 1)$ & 807.5024 & 807.5018 & -0.7 & 16:0-16:1 & $\mathrm{C}_{41} \mathrm{H}_{76} \mathrm{O}_{13} \mathrm{P}$ \\
\hline $\operatorname{PI}(34: 1)$ & 835.5318 & 835.5302 & -1.9 & 16:0-18:1 & $\mathrm{C}_{43} \mathrm{H}_{80} \mathrm{O}_{13} \mathrm{P}$ \\
\hline \multicolumn{6}{|c|}{ PI-Cer identified as $[\mathrm{M}-\mathrm{H}]^{-}$} \\
\hline PI-Cer(d18:1/14:0) & 750.4921 & 750.4921 & 0.0 & * & $\mathrm{C}_{38} \mathrm{H}_{73} \mathrm{NO}_{11} \mathrm{P}$ \\
\hline
\end{tabular}

Table 3. Phospholipid species identified in the total lipid extract of Chlorococcum amblystomatis by HILICHR-ESI-MS and MS/MS. $C$ number of carbon atoms, $N$ number of double bonds. ${ }^{\star}$ Identified based on the polar head fragmentation, calculated mass, and retention time. ${ }^{* *}$ Identified according to the calculated mass and the retention time.

The most abundant species in each class of glycolipids were: MGDG (34:7), identified as MGDG (16:4-18:3) and MGDG (18:4-16:3); MGMG (16:4); DGDG (34:3), identified as DGDG (16:0-18:3) and DGDG (18:1-16:2); DGMG (18:3); and SQDG (32:1), identified as SQDG (16:0-16:1). The only identified glycolipid species belonging to the SQMG class was identified by accurate mass measurements and by the presence on the MS/MS spectrum of the product ion corresponding to the polar head at $m / z 225$. Among the MGMG and SQDG species identified, we observed three oxidized species, MGMG (16:4-OH), SQDG (34:3-OH) identified as SQDG (18:3$\mathrm{OH}-16: 0)$ and SQDG (34:4-OH) identified as SQDG (18:4-OH-16:0). HILIC-HR-ESI-MS/MS analysis of all oxidized GL species suggested that a hydroxyl group was present in the fatty acyl group (Supplementary Table S3).

The relative quantification of the identified species was carried out as described in the methods section. Figure 6 shows the most abundant lipid species, identified as molecular ions, in the polar lipidome of Chlorococcum 
(A) Lysophosphatidylethanolamine as $[\mathrm{M}+\mathrm{H}]^{+}$

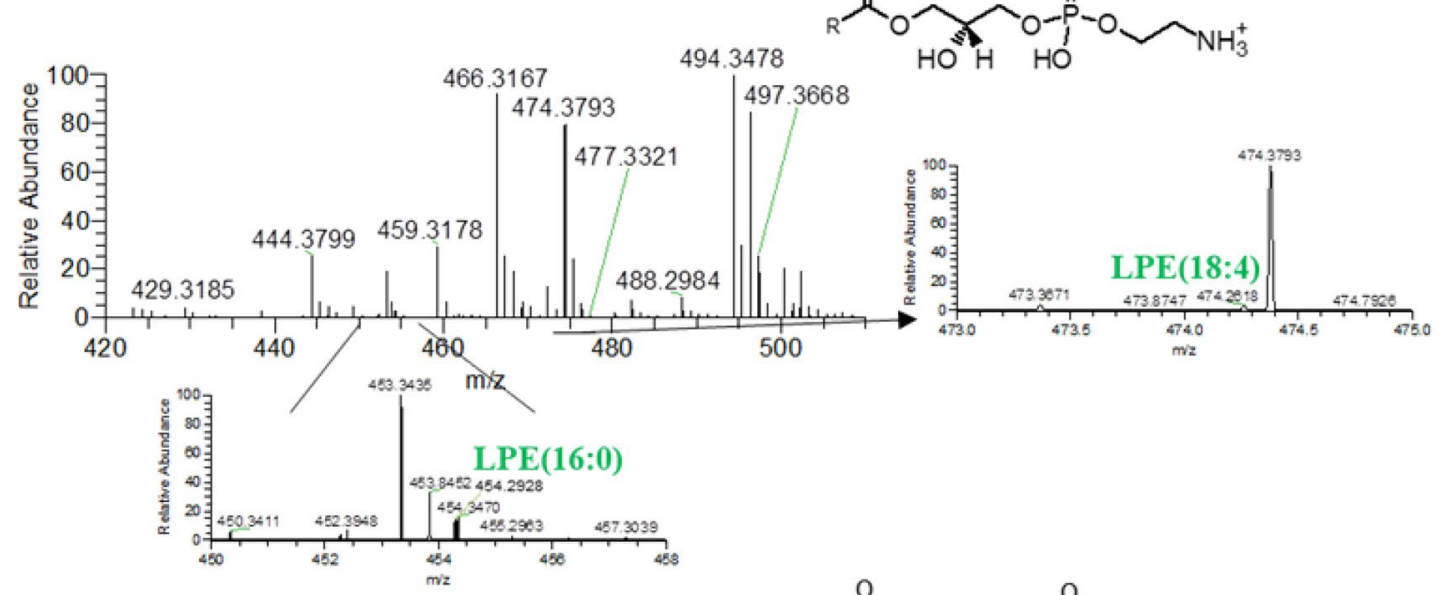

(B) Phosphatidylethanolamine as $[\mathrm{M}+\mathrm{H}]^{+}$

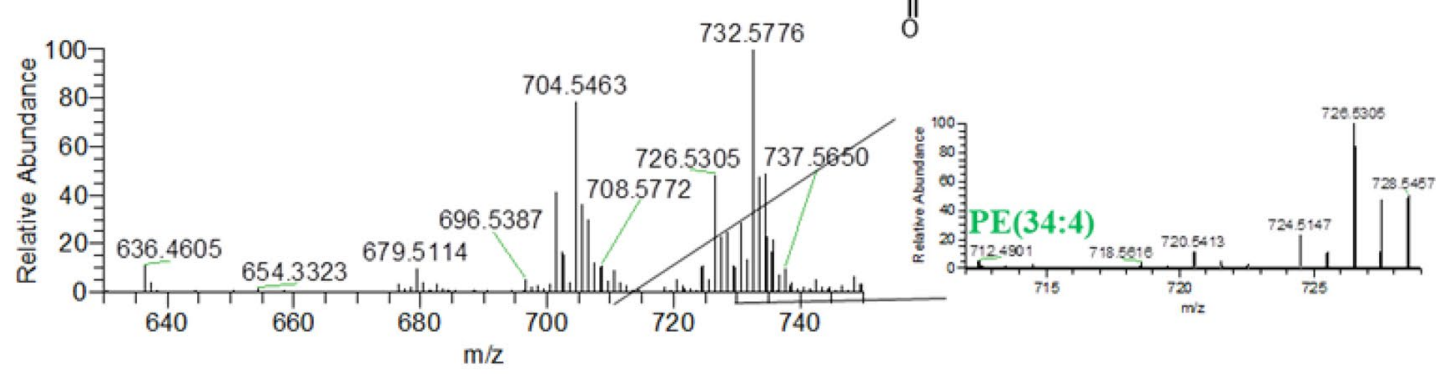

(C) Lysophosphatidylcholine as $[\mathrm{M}+\mathrm{H}]^{+}$

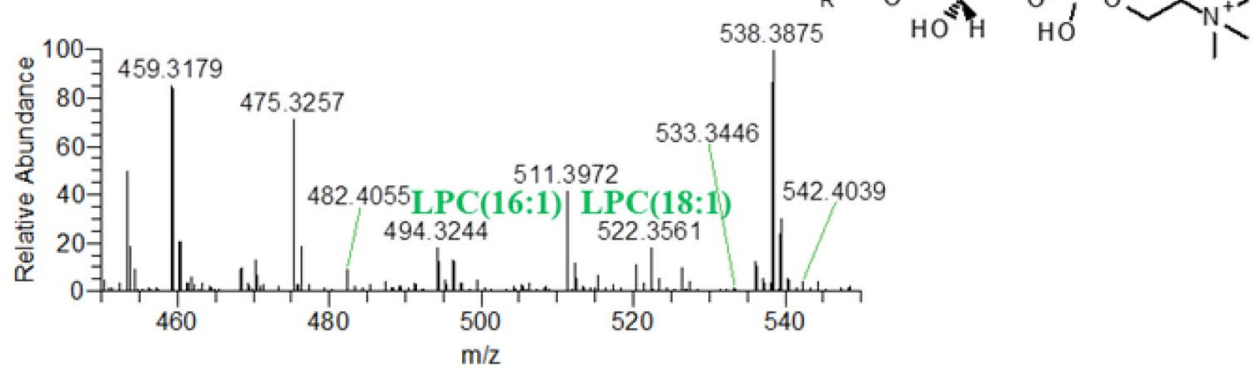

(D) Phosphatidylcholine as $[\mathrm{M}+\mathrm{H}]^{+}$

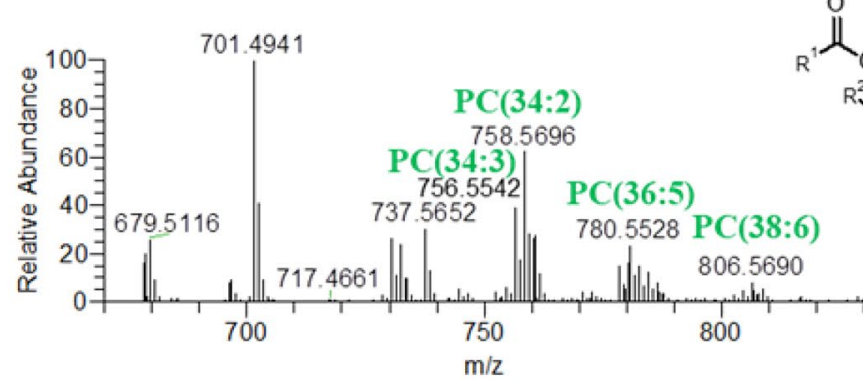<smiles>[R]C(=O)OC[C@@H]([CH])OC[C@H]([2H])OP(=O)(O)OCC[N+](C)(C)C</smiles>

Figure 1. HILIC-HR-ESI-MS spectra of the classes of phospholipids (PL) identified in positive ion mode as $[\mathrm{M}+\mathrm{H}]^{+}$. These classes were (A) lysophosphatidylethanolamine (LPE), (B) phosphatidylethanolamine (PE), (C) lysophosphatidylcholine (LPC) and (D) phosphatidylcholine (PC). 


\section{(A) Phosphatidylinositol as [M-H]}
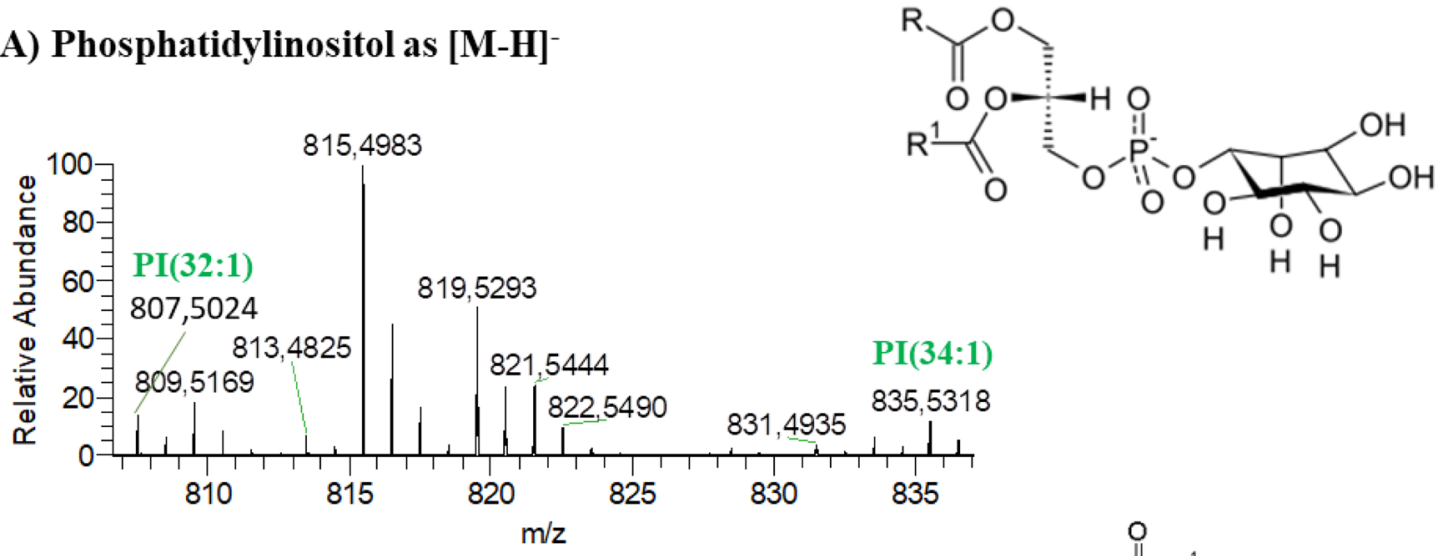

(B) Phosphatidyglycerol as [M-H $]^{-}$

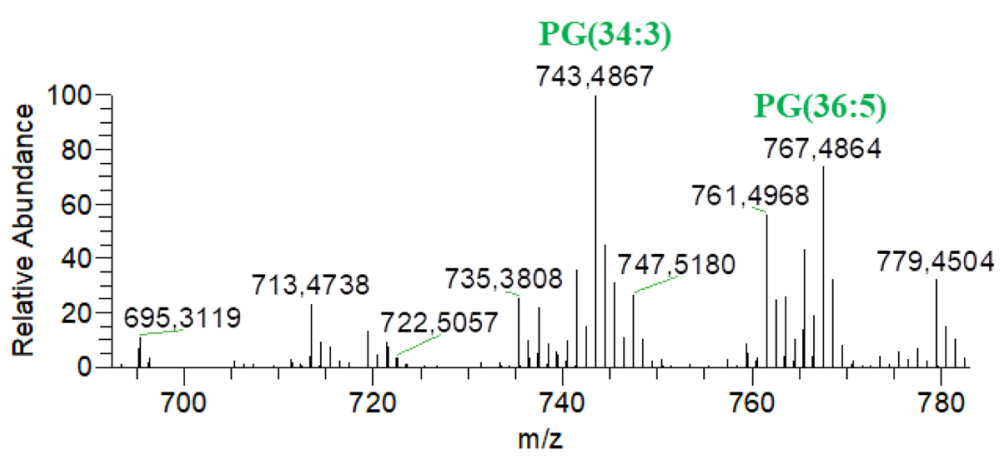

Figure 2. HILIC-HR-ESI-MS spectra of classes of phospholipids (PL) identified in negative ion mode as $[\mathrm{M}-\mathrm{H}]^{-}$. These classes were (A) phosphatidylinositol (PI) and (B) phosphatidylglycerol (PG).

amblystomatis, with a relative amount greater than or equal to $1.5 \%$. Glycolipids were the group of lipids with the largest number of species (101). The most abundant species was MGDG (34:7), assigned as MGDG (16:3-18:4) and MGDG (16:4-18:3), and the second most abundant species was SQDG (16:0-16:1).

LC-MS data was acquired using an internal standard for each class of phospholipids. LC-MS data were normalized against their assigned internal standard, to semi-quantify each molecular ion. Due to the lack of commercially available GL and BL standards, internal standards from classes with a retention time closer to those identified for GL and BL were used to normalize their respective data, as performed in our laboratory $\mathrm{y}^{45,52-54}$. The use of one internal standard is acceptable for semi-quantification because the ionization efficiency of polar lipids depends mainly on the polar head, while the length of the chain and the degree of unsaturation contribute little ${ }^{55,56}$. However, we would like to point out the limitations of our inter-class quantitation approach, because despite the similar retention time, the response factors for each class, even between phospholipids, tend to differ in ESI-MS, which prevents a robust and accurate quantification ${ }^{51-53}$. Normalized data were then used to semi-quantify the abundances of the identified molecular ions. For a clearer overview of the composition of the polar lipidome of Chlorococcum amblystomatis, we have summed up the relative percentage of all the detected lipid species belonging to glycolipids, betaines and phospholipids (Fig. 7A). According to the largest number of molecular ions, the highest cumulative levels were observed for glycolipids (51.9\%), followed by betaine lipids (30.1\%) and phospholipids (17.9\%). The sum of the relative percentage of all lipid species belonging to each class of lipids was also calculated. The highest cumulative levels were observed for DGTS (27.6\%) followed by SQDG (23.7\%) (Fig. 7B).

Evaluation of in vitro anti-inflammatory and antioxidant properties of Chlorococcum amblystomatis lipid extracts. The anti-inflammatory potential of lipid extracts from Chlorococcum amblystomatis were evaluated using a test kit for screening for inhibition of human COX-2. With $10 \mu \mathrm{l}$ of an extract of $50 \mu \mathrm{g} \mathrm{mL} \mathrm{L}^{-1}(5 \mu \mathrm{g})$, we have observed an inhibition of $87.5 \pm 0.1$ of COX-2 activity (Fig. 8). These results suggest that the lipid extracts of Chlorococcum amblystomatis have anti-inflammatory potential.

The antioxidant potential of lipid extracts from Chlorococcum amblystomatis were evaluated using free radical $\mathrm{DPPH}^{\bullet}$ and $\mathrm{ABTS}^{-+}$scavenging assays (Fig. 9), as described in the methods section. The results obtained for the $\mathrm{DPPH}$ assay revealed that the concentration of the extract resulting in a $40 \%$ inhibition (IC40) of $\mathrm{DPPH}^{*}$ was at an estimated concentration of $226.81 \pm 2.99 \mu \mathrm{g} \mathrm{mL}^{-1}$. The average antioxidant activity (Trolox equivalent, TE) was $76.25 \pm 1.02$ Trolox $\mu \mathrm{mol} \mathrm{g}{ }^{-1}$ of lipid extract. In contrast, the lipid extract which resulted in a $50 \%$ inhibition 


\begin{tabular}{|c|c|c|c|c|c|}
\hline Lipid species (C:N) & Calculated $m / z$ & Observed $m / z$ & Error $(\mathrm{ppm})$ & Fatty acyl chains (C:N) & Formula \\
\hline \multicolumn{6}{|c|}{ MGTS identified as $[\mathrm{M}+\mathrm{H}]^{+}$} \\
\hline MGTS(14:0) & 446.3482 & 446.3479 & -0.6 & $14: 0$ & $\mathrm{C}_{24} \mathrm{H}_{48} \mathrm{O}_{6} \mathrm{~N}$ \\
\hline MGTS(14:1) & 444.3325 & 444.3323 & -0.5 & 14:1 & $\mathrm{C}_{24} \mathrm{H}_{46} \mathrm{O}_{6} \mathrm{~N}$ \\
\hline MGTS(16:0) & 474.3795 & 474.3793 & -0.4 & $16: 0$ & $\mathrm{C}_{26} \mathrm{H}_{52} \mathrm{O}_{6} \mathrm{~N}$ \\
\hline $\operatorname{MGTS}(16: 1)$ & 472.3638 & 472.3635 & -0.7 & $16: 1$ & $\mathrm{C}_{26} \mathrm{H}_{50} \mathrm{O}_{6} \mathrm{~N}$ \\
\hline MGTS(16:2) & \begin{tabular}{|l|l|}
470.3482 \\
\end{tabular} & \begin{tabular}{|l|l|}
470.3485 \\
\end{tabular} & 0.7 & $16: 2$ & $\mathrm{C}_{26} \mathrm{H}_{48} \mathrm{O}_{6} \mathrm{~N}$ \\
\hline $\operatorname{MGTS}(16: 3)$ & 468.3325 & 468.3335 & 2.1 & ** & $\mathrm{C}_{26} \mathrm{H}_{46} \mathrm{O}_{6} \mathrm{~N}$ \\
\hline MGTS(16:4) & 466.3169 & 466.3167 & -0.4 & $16: 4$ & $\mathrm{C}_{26} \mathrm{H}_{44} \mathrm{O}_{6} \mathrm{~N}$ \\
\hline MGTS(18:0) & 502.4108 & 502.4115 & 1.5 & 18:0 & $\mathrm{C}_{28} \mathrm{H}_{56} \mathrm{O}_{6} \mathrm{~N}$ \\
\hline MGTS(18:1) & 500.3951 & 500.3948 & -0.6 & $18: 1$ & $\mathrm{C}_{28} \mathrm{H}_{54} \mathrm{O}_{6} \mathrm{~N}$ \\
\hline MGTS(18:2) & 498.3795 & 498.3789 & -1.1 & ** & $\mathrm{C}_{28} \mathrm{H}_{52} \mathrm{O}_{6} \mathrm{~N}$ \\
\hline $\operatorname{MGTS}(18: 3)$ & 496.3638 & 496.3636 & -0.4 & $* *$ & $\mathrm{C}_{28} \mathrm{H}_{50} \mathrm{O}_{6} \mathrm{~N}$ \\
\hline MGTS(18:4) & 494.3482 & 494.3478 & -0.7 & $18: 4$ & $\mathrm{C}_{28} \mathrm{H}_{48} \mathrm{O}_{6} \mathrm{~N}$ \\
\hline MGTS(18:5) & 492.3325 & 492.3318 & -1.5 & $18: 5$ & $\mathrm{C}_{28} \mathrm{H}_{46} \mathrm{O}_{6} \mathrm{~N}$ \\
\hline MGTS(20:0) & 530.4421 & 530.4402 & -3.5 & $20: 0$ & $\mathrm{C}_{30} \mathrm{H}_{60} \mathrm{O}_{6} \mathrm{~N}$ \\
\hline MGTS(20:4) & 522.3795 & \begin{tabular}{|l|l|}
522.3798 \\
\end{tabular} & 0.6 & $* *$ & $\mathrm{C}_{30} \mathrm{H}_{52} \mathrm{O}_{6} \mathrm{~N}$ \\
\hline $\operatorname{MGTS}(20: 5)$ & 520.3638 & 520.3637 & -0.2 & $20: 5$ & $\mathrm{C}_{30} \mathrm{H}_{50} \mathrm{O}_{6} \mathrm{~N}$ \\
\hline \multicolumn{6}{|c|}{ DGTS identified as $[\mathrm{M}+\mathrm{H}]^{+}$} \\
\hline DGTS(28:1) & 654.5309 & 654.5325 & 2.4 & * & $\mathrm{C}_{38} \mathrm{H}_{72} \mathrm{O}_{7} \mathrm{~N}$ \\
\hline DGTS(30:0) & 684.5778 & 684.5759 & -2.8 & $16: 0-14: 0$ & $\mathrm{C}_{40} \mathrm{H}_{78} \mathrm{O}_{7} \mathrm{~N}$ \\
\hline DGTS(30:1) & 682.5622 & 682.5619 & -0.4 & 16:1-14:0 & $\mathrm{C}_{40} \mathrm{H}_{76} \mathrm{O}_{7} \mathrm{~N}$ \\
\hline DGTS(30:2) & 680.5465 & 680.5444 & -3.1 & $16: 2-14: 0$ & $\mathrm{C}_{40} \mathrm{H}_{74} \mathrm{O}_{7} \mathrm{~N}$ \\
\hline DGTS(30:3) & 678.5309 & 678.5306 & -0.4 & ** & $\mathrm{C}_{40} \mathrm{H}_{72} \mathrm{O}_{7} \mathrm{~N}$ \\
\hline DGTS(30:4) & 676.5152 & 676.5151 & -0.1 & $16: 4-16: 0$ & $\mathrm{C}_{40} \mathrm{H}_{70} \mathrm{O}_{7} \mathrm{~N}$ \\
\hline DGTS(30:5) & 674.4996 & 674.5002 & 0.9 & $16: 4-14: 1$ & $\mathrm{C}_{40} \mathrm{H}_{68} \mathrm{O}_{7} \mathrm{~N}$ \\
\hline DGTS(32:0) & \begin{tabular}{|l|l|}
712.6091 \\
\end{tabular} & \begin{tabular}{|l|l|}
712.6067 \\
\end{tabular} & -3.4 & $16: 0-16: 0$ & $\mathrm{C}_{42} \mathrm{H}_{82} \mathrm{O}_{7} \mathrm{~N}$ \\
\hline DGTS(32:1) & 710.5935 & 710.5933 & -0.3 & $16: 1-16: 0$ & $\mathrm{C}_{42} \mathrm{H}_{80} \mathrm{O}_{7} \mathrm{~N}$ \\
\hline DGTS(32:2) & 708.5778 & 708.5772 & -0.8 & $16: 0-16: 2$ and $16: 1-16: 1$ & $\mathrm{C}_{42} \mathrm{H}_{78} \mathrm{O}_{7} \mathrm{~N}$ \\
\hline DGTS(32:3) & 706.5622 & 706.5610 & -1.7 & $16: 3-16: 0$ & $\mathrm{C}_{42} \mathrm{H}_{76} \mathrm{O}_{7} \mathrm{~N}$ \\
\hline DGTS(32:4) & 704.5465 & 704.5464 & -0.1 & $14: 0-18: 4,16: 4-16: 0$ and $16: 3-16: 1$ & $\mathrm{C}_{42} \mathrm{H}_{74} \mathrm{O}_{7} \mathrm{~N}$ \\
\hline DGTS(32:5) & 702.5309 & 702.5306 & -0.4 & ** & $\mathrm{C}_{42} \mathrm{H}_{72} \mathrm{O}_{7} \mathrm{~N}$ \\
\hline DGTS(32:6) & 700.5152 & 700.5152 & 0.0 & $16: 4-16: 2$ and $16: 3-16: 3$ & $\mathrm{C}_{42} \mathrm{H}_{70} \mathrm{O}_{7} \mathrm{~N}$ \\
\hline DGTS(32:7) & 698.4996 & 698.4993 & -0.4 & $16: 4-16: 3$ & $\mathrm{C}_{42} \mathrm{H}_{68} \mathrm{O}_{7} \mathrm{~N}$ \\
\hline DGTS(32:8) & 696.4839 & 696.4844 & 0.7 & $16: 4-16: 4$ & $\mathrm{C}_{42} \mathrm{H}_{66} \mathrm{O}_{7} \mathrm{~N}$ \\
\hline DGTS(34:1) & \begin{tabular}{|l|l|}
738.6248 \\
\end{tabular} & \begin{tabular}{|l|l|}
738.6251 \\
\end{tabular} & 0.4 & $16: 0-18: 1$ & $\mathrm{C}_{44} \mathrm{H}_{84} \mathrm{O}_{7} \mathrm{~N}$ \\
\hline DGTS(34:2) & 736.6091 & 736.6056 & -4.8 & $16: 1-18: 1,16: 0-18: 2$ and $20: 2-14: 0$ & $\mathrm{C}_{44} \mathrm{H}_{82} \mathrm{O}_{7} \mathrm{~N}$ \\
\hline DGTS(34:3) & 734.5935 & 734.5929 & -0.8 & $16: 2-18: 1,16: 1-18: 2$ and $20: 3-14: 0$ & $\mathrm{C}_{44} \mathrm{H}_{80} \mathrm{O}_{7} \mathrm{~N}$ \\
\hline DGTS(34:4) & 732.5778 & 732.5776 & -0.3 & $18: 4-16: 0$ and $18: 3-16: 1$ & $\mathrm{C}_{44} \mathrm{H}_{78} \mathrm{O}_{7} \mathrm{~N}$ \\
\hline DGTS(34:5) & 730.5622 & 730.5616 & -0.8 & $18: 4-16: 1,18: 1-16: 4$ and $18: 2-16: 3$ & $\mathrm{C}_{44} \mathrm{H}_{76} \mathrm{O}_{7} \mathrm{~N}$ \\
\hline DGTS(34:5-OH) & 746.5571 & 746.5575 & 0.5 & 16:4-OH-18:1, 16:1-OH-18:4 and 16:0-OH-18:5 & $\mathrm{C}_{44} \mathrm{H}_{76} \mathrm{O}_{8} \mathrm{~N}$ \\
\hline DGTS(34:6) & 728.5465 & 728.5458 & -1.0 & ** & $\mathrm{C}_{44} \mathrm{H}_{74} \mathrm{O}_{7} \mathrm{~N}$ \\
\hline DGTS(34:7) & 726.5309 & 726.5306 & -0.4 & $16: 4-18: 3$ and $16: 3-18: 4$ & $\mathrm{C}_{44} \mathrm{H}_{72} \mathrm{O}_{7} \mathrm{~N}$ \\
\hline DGTS(34:7-OH) & 742.5258 & 742.5258 & 0.0 & 16:4-OH-18:3 and 16:3-OH-18:4 & $\mathrm{C}_{44} \mathrm{H}_{72} \mathrm{O}_{8} \mathrm{~N}$ \\
\hline DGTS(34:8) & 724.5152 & 724.5149 & -0.5 & $16: 4-18: 4$ & $\mathrm{C}_{44} \mathrm{H}_{70} \mathrm{O}_{7} \mathrm{~N}$ \\
\hline DGTS(36:10) & \begin{tabular}{|l|l|}
748.5152 \\
\end{tabular} & 748.5140 & -1.6 & $18: 5-18: 5$ & $\mathrm{C}_{46} \mathrm{H}_{70} \mathrm{O}_{7} \mathrm{~N}$ \\
\hline DGTS(36:4) & 760.6091 & 760.6098 & 0.9 & $\begin{array}{l}\text { 16:0-20:4, 18:3-18:1, 18:2-18:2, 20:3-16:1, } \\
20: 2-16: 2,20: 1-16: 3 \text { and } 20: 0-16: 4\end{array}$ & $\mathrm{C}_{46} \mathrm{H}_{82} \mathrm{O}_{7} \mathrm{~N}$ \\
\hline DGTS(36:5) & 758.5935 & 758.5933 & -0.3 & $\begin{array}{l}18: 4-18: 1,20: 5-16: 0,20: 4-16: 1,20: 3-16: 2 \\
20: 2-16: 3,20: 1-16: 4 \text { and } 16: 1-20: 4\end{array}$ & $\mathrm{C}_{46} \mathrm{H}_{80} \mathrm{O}_{7} \mathrm{~N}$ \\
\hline DGTS(36:6) & \begin{tabular}{|l|l|}
756.5778 \\
\end{tabular} & \begin{tabular}{|l|l|}
756.5775 \\
\end{tabular} & -0.4 & $16: 1-20: 5,20: 3-16: 3,18: 2-18: 4$ and $18: 3-18: 3$ & $\mathrm{C}_{46} \mathrm{H}_{78} \mathrm{O}_{7} \mathrm{~N}$ \\
\hline DGTS(36:7) & 754.5622 & 754.5621 & -0.1 & $18: 4-18: 3$ & $\mathrm{C}_{46} \mathrm{H}_{76} \mathrm{O}_{7} \mathrm{~N}$ \\
\hline DGTS(36:8) & 752.5465 & 752.5462 & -0.4 & $18: 4-18: 4$ & $\mathrm{C}_{46} \mathrm{H}_{74} \mathrm{O}_{7} \mathrm{~N}$ \\
\hline DGTS(36:9) & 750.5309 & 750.5281 & -3.7 & $18: 5-18.4$ & $\mathrm{C}_{46} \mathrm{H}_{72} \mathrm{O}_{7} \mathrm{~N}$ \\
\hline DGTS(38:10) & \begin{tabular}{|l|l|}
776.5465 \\
\end{tabular} & \begin{tabular}{|l|l|}
776.5464 \\
\end{tabular} & -0.2 & $20: 5-18: 5$ & $\mathrm{C}_{48} \mathrm{H}_{74} \mathrm{O}_{7} \mathrm{~N}$ \\
\hline DGTS(38:7) & \begin{tabular}{|l}
782.5935 \\
\end{tabular} & \begin{tabular}{|l|l|}
782.5929 \\
\end{tabular} & -0.8 & $20: 5-18: 2$ & $\mathrm{C}_{48} \mathrm{H}_{80} \mathrm{O}_{7} \mathrm{~N}$ \\
\hline DGTS(38:8) & \begin{tabular}{|l|l|}
780.5778 \\
\end{tabular} & \begin{tabular}{|l|l|}
780.5743 \\
\end{tabular} & -4.5 & $20: 5-18: 3$ & $\mathrm{C}_{48} \mathrm{H}_{78} \mathrm{O}_{7} \mathrm{~N}$ \\
\hline DGTS(40:10) & \begin{tabular}{|l|}
804.5778 \\
\end{tabular} & \begin{tabular}{|l|}
804.5772 \\
\end{tabular} & -0.7 & $20: 5-20: 5$ & $\mathrm{C}_{50} \mathrm{H}_{78} \mathrm{O}_{7} \mathrm{~N}$ \\
\hline DGTS(40:9) & 806.5935 & 806.5905 & -3.7 & $20: 4-20: 5$ & $\mathrm{C}_{50} \mathrm{H}_{80} \mathrm{O}_{7} \mathrm{~N}$ \\
\hline
\end{tabular}


Table 4. Betaine lipids identified in the polar lipidome of Chlorococcum amblystomatis by HILIC-HR-ESIMS and MS/MS. $C$ number of carbon atoms, $N$ number of double bonds. ${ }^{\star}$ Identified based on the polar head fragmentation, calculated mass, and retention time. ${ }^{* *}$ Identified according to the calculated mass and the retention time.

(A) Diacylglycerol-trimethylhomoserine as $[\mathrm{M}+\mathrm{H}]^{+}$

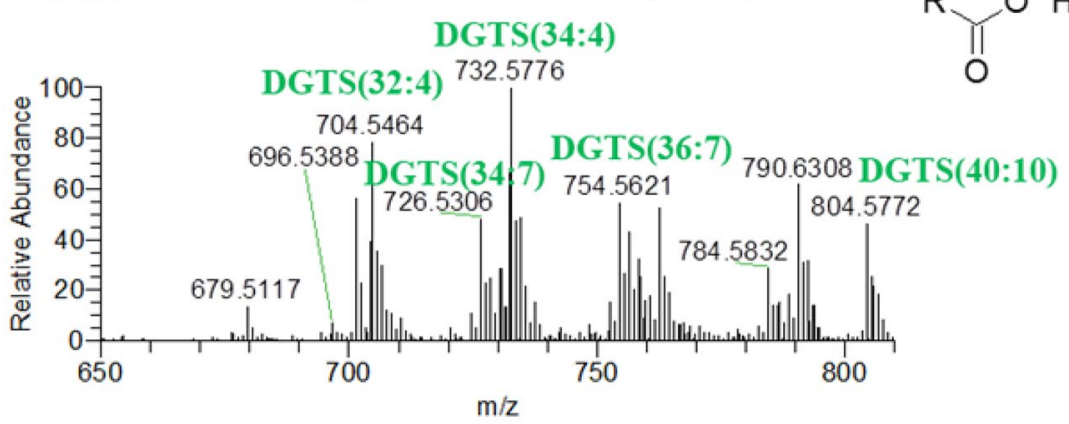<smiles>[R]C(=O)OCCCOCCC(C(=O)O)[N+](C)(C)C</smiles>

(B) Monocylglycerol-trimethylhomoserine as $[\mathrm{M}+\mathbf{H}]^{+}$

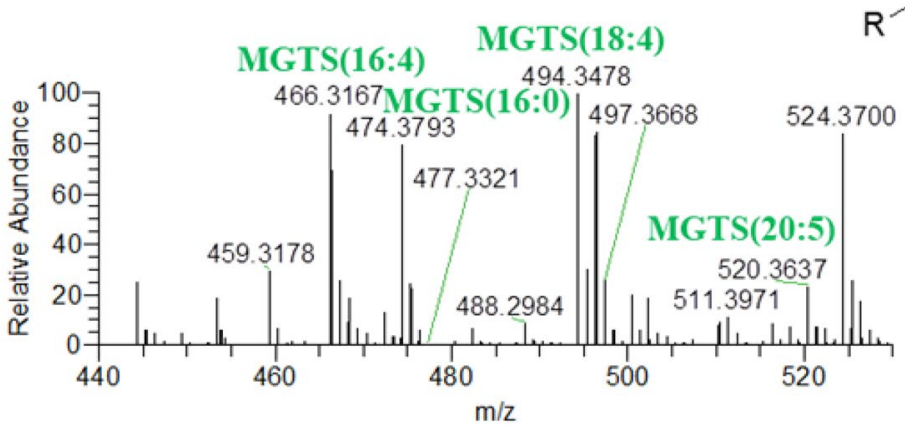<smiles>[R]C(=O)OC[C@@H](O)COCCC(C(=O)O)[N+](C)(C)C</smiles>

Figure 3. HILIC-HR-ESI-MS spectra of the classes of betaine lipids (BL) identified in positive ion mode as $[\mathrm{M}+\mathrm{H}]^{+}$. These classes were (A) diacylglycerol-trimethylhomoserine (DGTS) and (B) monoacylglyceroltrimethylhomoserine (MGTS).

(IC50) of ABTS ${ }^{\bullet+}$ was $37.69 \pm 4.44 \mu \mathrm{g} \mathrm{mL}^{-1}$ and the expressed TE activity of $428.81 \pm 54.32$ Trolox $\mu \mathrm{mol} \mathrm{g}^{-1}$ of lipid extract.

\section{Discussion}

Chlorococcum amblystomatis is a green microalga with great potential to be a sustainable resource of commercially important essential lipids $s^{1,2,29}$, with promising applications in several industrial sectors ${ }^{32,33}$. In our work, the lipid extracts represented $20.77 \pm 0.57 \%$, of the dry weight of the biomass, which is consistent with the lipid content reported for Chlorococcum $(8.71-32.3 \%)^{10,11,18-20}$. This wide range of lipid content determined by different studies is consistent with the variation in lipid content due to growing conditions $\mathrm{s}^{32,36,38-40}$ and the use of different extraction methods ${ }^{54}$.

The FA profile identified by GC-MS included as the most abundant FA: C16:0, C18:3 (n-3), C18:0, C16:4 (n-3), C20:5 (n-3) and C16:1-9, representing respectively 23\%, 19\%, 14\%, 11\%, 9\% and 7\% of the total FA pool. It is important to note that this is the first time that FA C16:4 (n-3) and C20:5 (n-3) have been reported in Chlorococcum amblystomatis. The values of relative abundance collected in the present work are different from those reported in the literature to Chlorococcum $\mathrm{sp}^{32,33,38,39}$. Such differences could result from differences in growth conditions ${ }^{55}$, different strains, different species, different derivatization methods or even GC detectors (FID or MS $)^{56}$. Chlorococcum amblystomatis extracts were rich in omega-3 PUFAs, representing $43.2 \%$, with a high contribution of the most abundant FA, C18:3 (n-3), C16:4 (n-3) and C20:5 (n-3). Omega-3 PUFAs are known to have a variety of health benefits, such as preventing chronic diseases, as they are associated with anti-inflammatory and antioxidant protection, benefits for the cardiovascular system, prevention of breast cancer, improvement of neurological capacities and visual development ${ }^{57-59}$. To understand the beneficial impact on health and assess the nutritional quality of Chlorococcum amblystomatis, we calculated the values of AI (0.5), TI (0.3), h/H (1.4), 
(A) Monogalactosylmonoacylglycerol as $\left[\mathrm{M}+\mathrm{NH}_{4}\right]^{+}$

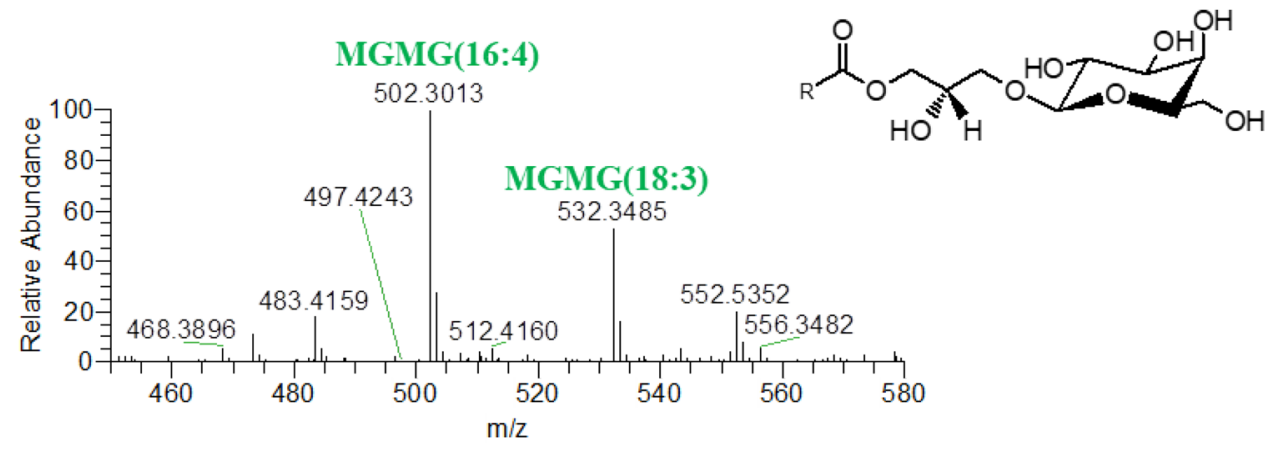

(B) Monogalactosyldiacylglycerol as $\left[\mathrm{M}+\mathrm{NH}_{4}\right]^{+}$

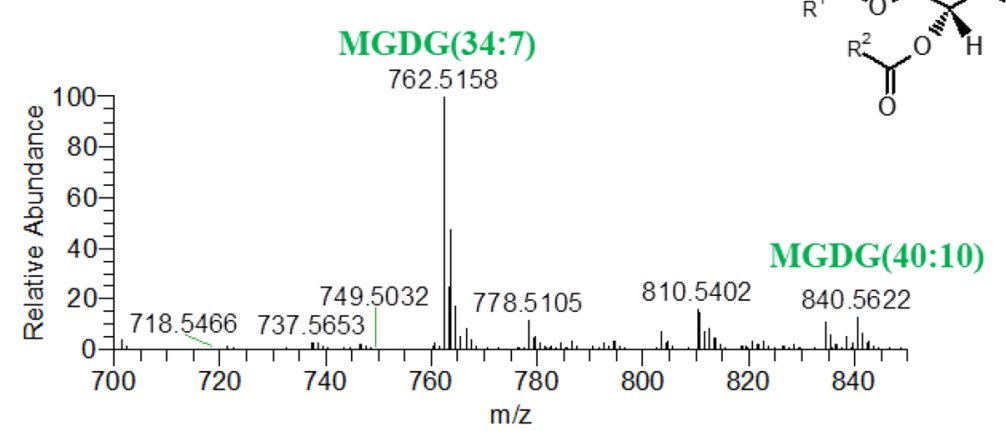

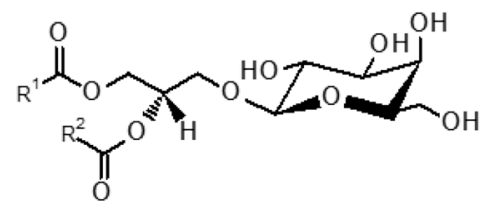

(C) Digalactosylmonoacylglycerol as $\left[\mathrm{M}+\mathrm{NH}_{4}\right]^{+}$
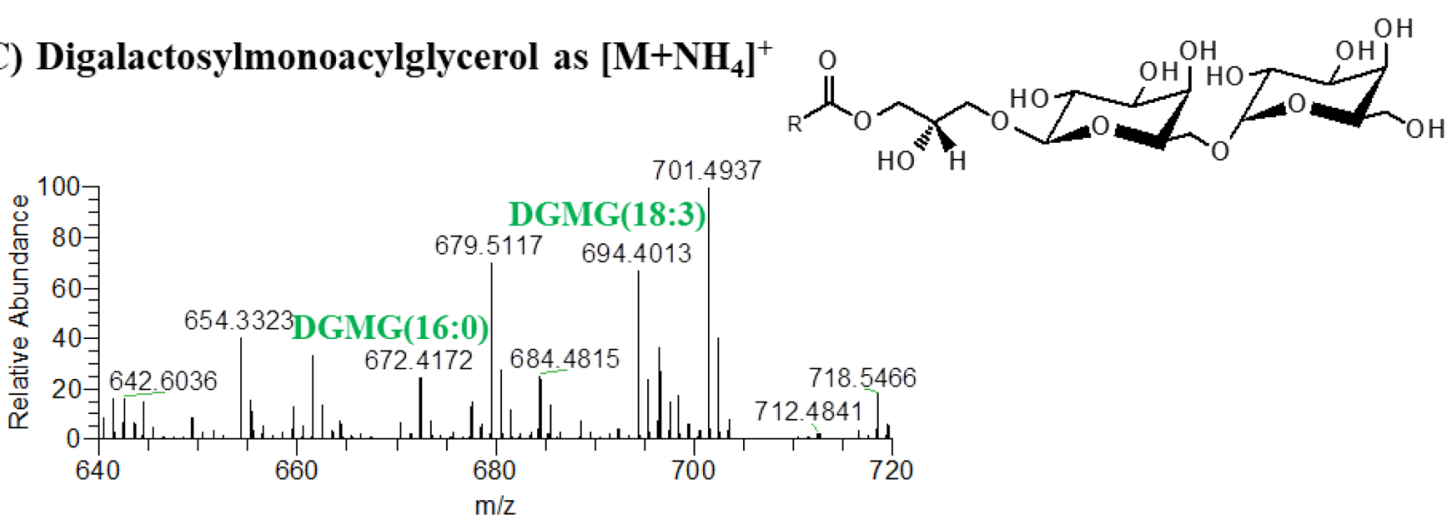

(D) Digalactosyldiacylglycerol as $\left[\mathrm{M}+\mathrm{NH}_{4}\right]^{+}$

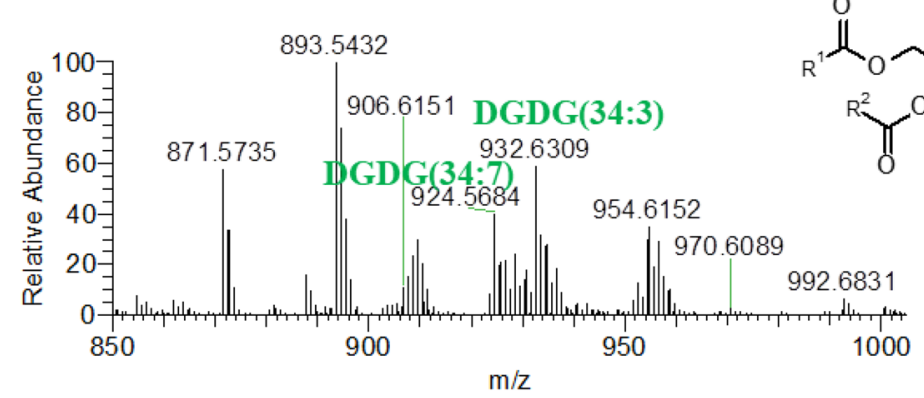

Figure 4. HILIC-HR-ESI-MS spectra of the classes of glycolipids (GL) identified in positive ion mode as $\left[\mathrm{M}+\mathrm{NH}_{4}\right]^{+}$. These classes were (A) monogalactosylmonoacylglycerol (MGMG), (B) monogalactosyldiacylglycerol (MGDG), (C) digalactosylmonoacylglycerol (DGMG) and (D) digalactosyldiacylglycerol (DGDG). 


\begin{tabular}{|c|c|c|c|c|c|}
\hline Lipid species (C:N) & Calculated $m / z$ & Observed $m / z$ & Error (ppm) & Fatty acyl chains (C:N) & Formula \\
\hline \multicolumn{6}{|c|}{ MGMG identified as $\left[\mathrm{M}+\mathrm{NH}_{4}\right]^{+}$} \\
\hline MGMG(14:0) & 482.3329 & 482.3324 & -1.1 & $14: 0$ & $\mathrm{C}_{23} \mathrm{H}_{48} \mathrm{NO}_{9}$ \\
\hline MGMG(16:0) & 510.3642 & 510.3639 & -0.6 & $16: 0$ & $\mathrm{C}_{25} \mathrm{H}_{52} \mathrm{NO}_{9}$ \\
\hline MGMG(16:1) & 508.3486 & 508.3482 & -0.7 & $16: 1$ & $\mathrm{C}_{25} \mathrm{H}_{50} \mathrm{NO}_{9}$ \\
\hline MGMG(16:3) & 504.3173 & 504.3174 & 0.3 & ** & $\mathrm{C}_{25} \mathrm{H}_{46} \mathrm{NO}_{9}$ \\
\hline MGMG(16:4) & 502.3016 & 502.3013 & -0.6 & $16: 4$ & $\mathrm{C}_{25} \mathrm{H}_{44} \mathrm{NO}_{9}$ \\
\hline MGMG(16:4-OH) & 518.2965 & 518.2963 & -0.4 & 16:4-OH & $\mathrm{C}_{25} \mathrm{H}_{44} \mathrm{NO}_{9} \mathrm{O}$ \\
\hline MGMG(18:1) & 536.3799 & 536.3793 & -1.0 & $18: 1$ & $\mathrm{C}_{27} \mathrm{H}_{54} \mathrm{NO}_{9}$ \\
\hline MGMG(18:3) & 532.3486 & 532.3485 & -0.1 & $18: 3$ & $\mathrm{C}_{27} \mathrm{H}_{50} \mathrm{NO}_{9}$ \\
\hline MGMG(18:4) & 530.3329 & 530.3330 & 0.2 & * & $\mathrm{C}_{27} \mathrm{H}_{48} \mathrm{NO}_{9}$ \\
\hline MGMG(20:4) & 558.3642 & 558.3636 & -1.1 & $* *$ & $\mathrm{C}_{29} \mathrm{H}_{52} \mathrm{NO}_{9}$ \\
\hline MGMG(20:5) & 556.3486 & 556.3482 & -0.6 & $* *$ & $\mathrm{C}_{29} \mathrm{H}_{50} \mathrm{NO}_{9}$ \\
\hline \multicolumn{6}{|c|}{ MGDG identified as $\left[\mathrm{M}+\mathrm{NH}_{4}\right]^{+}$} \\
\hline MGDG(30:0) & 720.5625 & 720.5593 & -4.4 & $* *$ & $\mathrm{C}_{39} \mathrm{H}_{78} \mathrm{NO}_{10}$ \\
\hline MGDG(30:1) & 718.5464 & 718.5466 & 0.3 & 16:1-14:0 & $\mathrm{C}_{39} \mathrm{H}_{76} \mathrm{NO}_{10}$ \\
\hline MGDG(32:0) & 748.5939 & 748.5910 & -3.8 & $16: 0-16: 0$ & $\mathrm{C}_{41} \mathrm{H}_{82} \mathrm{NO}_{10}$ \\
\hline MGDG(32:1) & 746.5777 & 746.5780 & 0.4 & $16: 1-16: 0$ & $\mathrm{C}_{41} \mathrm{H}_{80} \mathrm{NO}_{10}$ \\
\hline MGDG(32:2) & 744.5626 & 744.5624 & -0.3 & $16: 1-16: 1,14: 0-18: 2$ and $16: 0-16: 2$ & $\mathrm{C}_{41} \mathrm{H}_{78} \mathrm{NO}_{10}$ \\
\hline MGDG(32:3) & 742.5464 & 742.5467 & 0.4 & ** & $\mathrm{C}_{41} \mathrm{H}_{76} \mathrm{NO}_{10}$ \\
\hline MGDG(32:4) & 740.5307 & 740.5305 & -0.3 & $* *$ & $\mathrm{C}_{41} \mathrm{H}_{74} \mathrm{NO}_{10}$ \\
\hline MGDG(32:5) & 738.5156 & 738.5158 & 0.2 & $* *$ & $\mathrm{C}_{41} \mathrm{H}_{72} \mathrm{NO}_{10}$ \\
\hline MGDG(32:6) & 736.4994 & 736.4999 & 0.7 & ** & $\mathrm{C}_{41} \mathrm{H}_{70} \mathrm{NO}_{10}$ \\
\hline MGDG(32:7) & 734.4843 & 734.4835 & -1.1 & $* *$ & $\mathrm{C}_{41} \mathrm{H}_{68} \mathrm{NO}_{10}$ \\
\hline MGDG(32:8) & 732.4687 & 732.4686 & -0.1 & $16: 4-16: 4$ & $\mathrm{C}_{41} \mathrm{H}_{66} \mathrm{NO}_{10}$ \\
\hline MGDG(34:1) & 774.6090 & 774.6073 & -2.2 & ** & $\mathrm{C}_{43} \mathrm{H}_{84} \mathrm{NO}_{10}$ \\
\hline MGDG(34:2) & 772.5933 & 772.5926 & -0.9 & $18: 2-16: 0$ & $\mathrm{C}_{43} \mathrm{H}_{82} \mathrm{NO}_{10}$ \\
\hline MGDG(34:3) & 770.5782 & 770.5782 & 0.0 & $18: 3-16: 0$ and $18: 2-16: 1$ & $\mathrm{C}_{43} \mathrm{H}_{80} \mathrm{NO}_{10}$ \\
\hline MGDG(34:4) & 768.5626 & 768.5645 & 2.5 & $\begin{array}{l}\text { 16:2-18:2, 16:1-18:3, 16:0-18:4, 16:3-18:1 and } \\
16: 4-18: 0\end{array}$ & $\mathrm{C}_{43} \mathrm{H}_{78} \mathrm{NO}_{10}$ \\
\hline MGDG(34:5) & 766.5469 & 766.5474 & 0.6 & * & $\mathrm{C}_{43} \mathrm{H}_{76} \mathrm{NO}_{10}$ \\
\hline MGDG(34:7) & 762.5156 & 762.5158 & 0.2 & $16: 4-18: 3$ and $18: 4-16: 3$ & $\mathrm{C}_{43} \mathrm{H}_{72} \mathrm{NO}_{10}$ \\
\hline MGDG(34:8) & 760.5000 & 760.4999 & -0.1 & ** & $\mathrm{C}_{43} \mathrm{H}_{70} \mathrm{NO}_{10}$ \\
\hline MGDG(36:3) & 798.6095 & 798.6055 & -5.0 & $* *$ & $\mathrm{C}_{45} \mathrm{H}_{84} \mathrm{NO}_{10}$ \\
\hline MGDG(36:4) & 796.5933 & 796.5892 & -5.1 & ** & $\mathrm{C}_{45} \mathrm{H}_{82} \mathrm{NO}_{10}$ \\
\hline $\operatorname{MGDG}(36: 5)$ & 794.5782 & 794.5778 & -0.5 & $\begin{array}{l}18: 3-18: 2,18: 1-18: 4,20: 5-16: 0,16: 4-20: 2 \text { and } \\
16: 1-20: 4\end{array}$ & $\mathrm{C}_{45} \mathrm{H}_{80} \mathrm{NO}_{10}$ \\
\hline MGDG(36:6) & 792.5625 & 792.5623 & -0.3 & $18: 3-18: 3,16: 1-20: 5$ and $16: 4-20: 2$ & $\mathrm{C}_{45} \mathrm{H}_{78} \mathrm{NO}_{10}$ \\
\hline MGDG(36:7) & 790.5469 & 790.5470 & 0.1 & ** & $\mathrm{C}_{45} \mathrm{H}_{76} \mathrm{NO}_{10}$ \\
\hline MGDG(36:8) & 788.5313 & 788.5339 & 3.3 & ** & $\mathrm{C}_{45} \mathrm{H}_{74} \mathrm{NO}_{10}$ \\
\hline MGDG(38:6) & 820.5939 & 820.5931 & -1.0 & $18: 1-20: 5,18: 2-20: 4$ and $18: 3-20: 3$ & $\mathrm{C}_{47} \mathrm{H}_{82} \mathrm{NO}_{10}$ \\
\hline MGDG(38:7) & 818.5782 & 818.5777 & -0.6 & $20: 5-18: 2$ and $18: 3-20: 4$ & $\mathrm{C}_{47} \mathrm{H}_{80} \mathrm{NO}_{10}$ \\
\hline MGDG(38:8) & 816.5626 & 816.5614 & -1.4 & ** & $\mathrm{C}_{47} \mathrm{H}_{78} \mathrm{NO}_{10}$ \\
\hline MGDG(40:10) & 840.5626 & 840.5622 & -0.4 & $20: 5-20: 5$ & $\mathrm{C}_{49} \mathrm{H}_{78} \mathrm{NO}_{10}$ \\
\hline MGDG(40:8) & 844.5939 & 844.5916 & -2.7 & $20: 4-20: 4$ & $\mathrm{C}_{49} \mathrm{H}_{82} \mathrm{NO}_{10}$ \\
\hline MGDG(40:7) & 846.6095 & 846.6097 & 0.2 & ** & $\mathrm{C}_{49} \mathrm{H}_{84} \mathrm{NO}_{10}$ \\
\hline \multicolumn{6}{|c|}{ DGMG identified as $\left[\mathrm{M}+\mathrm{NH}_{4}\right]^{+}$} \\
\hline DGMG(14:0) & 644.3857 & 644.3848 & -1.4 & $* *$ & $\mathrm{C}_{29} \mathrm{H}_{58} \mathrm{NO}_{14}$ \\
\hline DGMG(16:0) & 672.4170 & 672.4172 & 0.3 & 16:0 & $\mathrm{C}_{31} \mathrm{H}_{62} \mathrm{NO}_{14}$ \\
\hline DGMG(16:1) & 670.4014 & 670.4012 & -0.3 & $16: 1$ & $\mathrm{C}_{31} \mathrm{H}_{60} \mathrm{NO}_{14}$ \\
\hline DGMG(16:2) & 668.3857 & 668.3854 & -0.5 & ** & $\mathrm{C}_{31} \mathrm{H}_{58} \mathrm{NO}_{14}$ \\
\hline DGMG(16:3) & 666.3701 & 666.3699 & -0.3 & $* *$ & $\mathrm{C}_{31} \mathrm{H}_{56} \mathrm{NO}_{14}$ \\
\hline DGMG(16:4) & 664.3544 & 664.3541 & -0.5 & ** & $\mathrm{C}_{31} \mathrm{H}_{54} \mathrm{NO}_{14}$ \\
\hline DGMG(18:1) & 698.4327 & 698.4327 & 0.0 & $18: 1$ & $\mathrm{C}_{33} \mathrm{H}_{64} \mathrm{NO}_{14}$ \\
\hline DGMG(18:2) & 696.4170 & 696.4149 & -3.0 & $* *$ & $\mathrm{C}_{33} \mathrm{H}_{62} \mathrm{NO}_{14}$ \\
\hline DGMG(18:3) & 694.4014 & 694.4013 & -0.1 & $18: 3$ & $\mathrm{C}_{33} \mathrm{H}_{60} \mathrm{NO}_{14}$ \\
\hline DGMG(18:4) & 692.3857 & 692.3856 & -0.2 & $* *$ & $\mathrm{C}_{33} \mathrm{H}_{58} \mathrm{NO}_{14}$ \\
\hline DGMG(20:5) & 718.4014 & 718.4009 & -0.7 & $* *$ & $\mathrm{C}_{35} \mathrm{H}_{60} \mathrm{NO}_{14}$ \\
\hline
\end{tabular}




\begin{tabular}{|c|c|c|c|c|c|}
\hline Lipid species (C:N) & Calculated $m / z$ & Observed $m / z$ & Error (ppm) & Fatty acyl chains (C:N) & Formula \\
\hline \multicolumn{6}{|c|}{ DGDG identified as $\left[\mathrm{M}+\mathrm{NH}_{4}\right]^{+}$} \\
\hline DGDG(30:0) & 882.6154 & 882.6111 & -4.9 & $* *$ & $\mathrm{C}_{45} \mathrm{H}_{88} \mathrm{O}_{15} \mathrm{~N}$ \\
\hline DGDG(30:1) & 880.5997 & 880.5990 & -0.8 & $16: 1-14: 0$ & $\mathrm{C}_{45} \mathrm{H}_{86} \mathrm{O}_{15} \mathrm{~N}$ \\
\hline DGDG(32:1) & 908.6310 & 908.6306 & -0.4 & $16: 0-16: 1$ & $\mathrm{C}_{47} \mathrm{H}_{90} \mathrm{O}_{15} \mathrm{~N}$ \\
\hline DGDG(32:2) & 906.6154 & 906.6151 & -0.3 & $16: 0-16: 2$ and $16: 1-16: 1$ & $\mathrm{C}_{47} \mathrm{H}_{88} \mathrm{O}_{15} \mathrm{~N}$ \\
\hline DGDG(32:3) & 904.5997 & 904.6011 & 1.5 & $18: 3-14: 0,16: 0-16: 3$ and $16: 1-16: 2$ & $\mathrm{C}_{47} \mathrm{H}_{86} \mathrm{O}_{15} \mathrm{~N}$ \\
\hline DGDG(32:4) & 902.5841 & 902.5846 & 0.6 & $18: 3-14: 1,16: 0-16: 4$ and $14: 0-18: 4$ & $\mathrm{C}_{47} \mathrm{H}_{84} \mathrm{O}_{15} \mathrm{~N}$ \\
\hline DGDG(32:5) & 900.5684 & 900.5678 & -0.7 & $16: 1-16: 4$ & $\mathrm{C}_{47} \mathrm{H}_{82} \mathrm{O}_{15} \mathrm{~N}$ \\
\hline DGDG(32:6) & 898.5528 & 898.5512 & -1.8 & ** & $\mathrm{C}_{47} \mathrm{H}_{80} \mathrm{O}_{15} \mathrm{~N}$ \\
\hline DGDG(34:1) & 936.6623 & 936.6615 & -0.9 & 18:1-16:0 & $\mathrm{C}_{49} \mathrm{H}_{94} \mathrm{O}_{15} \mathrm{~N}$ \\
\hline DGDG(34:2) & 934.6467 & 934.6443 & -2.6 & 18:1-16:1 and 18:2-16:0 & $\mathrm{C}_{49} \mathrm{H}_{92} \mathrm{O}_{15} \mathrm{~N}$ \\
\hline DGDG(34:3) & 932.6310 & 932.6309 & -0.1 & $16: 0-18: 3$ and $18: 1-16: 2$ & $\mathrm{C}_{49} \mathrm{H}_{90} \mathrm{O}_{15} \mathrm{~N}$ \\
\hline DGDG(34:4) & 930.6154 & 930.6143 & -1.2 & $\begin{array}{l}18: 1-16: 3,18: 2-16: 2,18: 3-16: 1,18: 4-16: 0 \text { and } \\
14: 0-20: 4\end{array}$ & $\mathrm{C}_{49} \mathrm{H}_{88} \mathrm{O}_{15} \mathrm{~N}$ \\
\hline $\operatorname{DGDG}(34: 5)$ & 928.5997 & 928.5991 & -0.6 & $\begin{array}{l}18: 3-16: 2,18: 2-16: 3,18: 1-16: 4,16: 1-18: 4 \text { and } \\
14: 0-20: 5\end{array}$ & $\mathrm{C}_{49} \mathrm{H}_{86} \mathrm{O}_{15} \mathrm{~N}$ \\
\hline DGDG(34:6) & 926.5841 & 926.5820 & -2.3 & $* *$ & $\mathrm{C}_{49} \mathrm{H}_{84} \mathrm{O}_{15} \mathrm{~N}$ \\
\hline DGDG(34:7) & 924.5684 & 924.5684 & 0.0 & $18: 3-16: 4$ and $18: 4-16: 3$ & $\mathrm{C}_{49} \mathrm{H}_{82} \mathrm{O}_{15} \mathrm{~N}$ \\
\hline DGDG(34:8) & 922.5528 & 922.5501 & -2.9 & ** & $\mathrm{C}_{49} \mathrm{H}_{80} \mathrm{O}_{15} \mathrm{~N}$ \\
\hline DGDG(36:2) & 962.6780 & 962.6751 & -3.0 & $* *$ & $\mathrm{C}_{51} \mathrm{H}_{96} \mathrm{O}_{15} \mathrm{~N}$ \\
\hline DGDG(36:3) & 960.6623 & 960.6606 & -1.8 & $18: 3-18: 0$ and $18: 1-18: 2$ & $\mathrm{C}_{51} \mathrm{H}_{94} \mathrm{O}_{15} \mathrm{~N}$ \\
\hline DGDG(36:4) & 958.6467 & 958.6433 & -3.5 & ** & $\mathrm{C}_{51} \mathrm{H}_{92} \mathrm{O}_{15} \mathrm{~N}$ \\
\hline DGDG(36:5) & 956.6310 & 956.6301 & -0.9 & $20: 5-16: 0,18: 1-18: 4$ and $20: 1-16: 4$ & $\mathrm{C}_{51} \mathrm{H}_{90} \mathrm{O}_{15} \mathrm{~N}$ \\
\hline DGDG(36:6) & 954.6154 & 954.6152 & -0.2 & $18: 3-18: 3,20: 5-16: 1$ and $18: 4-18: 2$ & $\mathrm{C}_{51} \mathrm{H}_{88} \mathrm{O}_{15} \mathrm{~N}$ \\
\hline DGDG(36:7) & 952.5997 & 952.5999 & 0.2 & $18: 3-18: 4$ & $\mathrm{C}_{51} \mathrm{H}_{86} \mathrm{O}_{15} \mathrm{~N}$ \\
\hline DGDG(36:8) & 950.5841 & 950.5835 & -0.6 & $18: 4-18: 4$ & $\mathrm{C}_{51} \mathrm{H}_{84} \mathrm{O}_{15} \mathrm{~N}$ \\
\hline DGDG(38:6) & 982.6467 & 982.6421 & -4.7 & $* *$ & $\mathrm{C}_{53} \mathrm{H}_{92} \mathrm{O}_{15} \mathrm{~N}$ \\
\hline DGDG(38:7) & 980.6310 & 980.6312 & 0.2 & $18: 2-20: 5$ & $\mathrm{C}_{53} \mathrm{H}_{90} \mathrm{O}_{15} \mathrm{~N}$ \\
\hline DGDG(40:10) & 1002.6154 & 1002.6148 & -0.6 & $20: 5-20: 5$ & $\mathrm{C}_{55} \mathrm{H}_{88} \mathrm{O}_{15} \mathrm{~N}$ \\
\hline DGDG(40:9) & 1004.6310 & 1004.6323 & 1.3 & ** & $\mathrm{C}_{55} \mathrm{H}_{90} \mathrm{O}_{15} \mathrm{~N}$ \\
\hline \multicolumn{6}{|c|}{ SQDG identified as $[\mathrm{M}-\mathrm{H}]^{-}$} \\
\hline SQDG(28:0) & 737.4510 & 737.4492 & -2.4 & $* *$ & $\mathrm{C}_{37} \mathrm{H}_{69} \mathrm{O}_{12} \mathrm{~S}$ \\
\hline SQDG(30:0) & 765.4823 & 765.4818 & -0.6 & $14: 0-16: 0$ & $\mathrm{C}_{39} \mathrm{H}_{73} \mathrm{O}_{12} \mathrm{~S}$ \\
\hline SQDG(30:1) & 763.4666 & 763.4667 & 0.1 & 14:0-16:1 & $\mathrm{C}_{39} \mathrm{H}_{71} \mathrm{O}_{12} \mathrm{~S}$ \\
\hline SQDG(32:0) & 793.5136 & 793.5135 & -0.1 & ** & $\mathrm{C}_{41} \mathrm{H}_{77} \mathrm{O}_{12} \mathrm{~S}$ \\
\hline SQDG(32:1) & 791.4979 & 791.4982 & 0.3 & $16: 0-16: 1$ & $\mathrm{C}_{41} \mathrm{H}_{75} \mathrm{O}_{12} \mathrm{~S}$ \\
\hline SQDG(32:2) & 789.4823 & 789.4821 & -0.2 & $16: 1-16: 1$ and $16: 0-16: 2$ & $\mathrm{C}_{41} \mathrm{H}_{73} \mathrm{O}_{12} \mathrm{~S}$ \\
\hline SQDG(32:3) & 787.4666 & 787.4668 & 0.2 & $16: 1-16: 2,16: 0-16: 3$ and $18: 1-14: 1$ & $\mathrm{C}_{41} \mathrm{H}_{71} \mathrm{O}_{12} \mathrm{~S}$ \\
\hline SQDG(32:4) & 785.4510 & 785.4517 & 0.9 & $16: 0-16: 4,16: 1-16: 3$ and $16: 2-16: 2$ & $\mathrm{C}_{41} \mathrm{H}_{69} \mathrm{O}_{12} \mathrm{~S}$ \\
\hline SQDG(34:0) & 821.5449 & 821.5446 & -0.3 & $16: 0-18: 0$ & $\mathrm{C}_{43} \mathrm{H}_{81} \mathrm{O}_{12} \mathrm{~S}$ \\
\hline SQDG(34:1) & 819.5292 & 819.5296 & 0.5 & $16: 0-18: 1$ & $\mathrm{C}_{43} \mathrm{H}_{79} \mathrm{O}_{12} \mathrm{~S}$ \\
\hline SQDG(34:3) & 815.4979 & 815.4982 & 0.3 & $16: 0-18: 3$ and $16: 2-18: 1$ & $\mathrm{C}_{43} \mathrm{H}_{75} \mathrm{O}_{12} \mathrm{~S}$ \\
\hline $\operatorname{SQDG}(34: 4)$ & 813.4823 & 813.4829 & 0.8 & $\begin{array}{l}\text { 16:0-18:4, 16:1-18:3, 16:2-18:2, 18:1-16:3 and } \\
14: 0-20: 4\end{array}$ & $\mathrm{C}_{43} \mathrm{H}_{73} \mathrm{O}_{12} \mathrm{~S}$ \\
\hline $\operatorname{SQDG}(34: 5)$ & 811.4666 & 811.4667 & 0.1 & $\begin{array}{l}\text { 16:0-18:5, 16:1-18:4, 16:2-18:3, 16:3-18:2 and } \\
16: 4-18: 1\end{array}$ & $\mathrm{C}_{43} \mathrm{H}_{71} \mathrm{O}_{12} \mathrm{~S}$ \\
\hline SQDG(36:0) & 849.5762 & 849.5761 & -0.1 & $16: 0-20: 0$ & $\mathrm{C}_{45} \mathrm{H}_{85} \mathrm{O}_{12} \mathrm{~S}$ \\
\hline SQDG(36:3) & 843.5292 & 843.5287 & -0.6 & ** & $\mathrm{C}_{45} \mathrm{H}_{79} \mathrm{O}_{12} \mathrm{~S}$ \\
\hline SQDG(36:4) & 841.5136 & 841.5135 & -0.1 & $16: 0-20: 4$ & $\mathrm{C}_{45} \mathrm{H}_{77} \mathrm{O}_{12} \mathrm{~S}$ \\
\hline SQDG(36:5) & 839.4979 & 839.4987 & 0.9 & ** & $\mathrm{C}_{45} \mathrm{H}_{75} \mathrm{O}_{12} \mathrm{~S}$ \\
\hline SQDG(36:6) & 837.4823 & 837.4823 & 0.0 & $16: 1-20: 5,20: 4-16: 2,18: 2-18: 4$ and $18: 3-18: 3$ & $\mathrm{C}_{45} \mathrm{H}_{73} \mathrm{O}_{12} \mathrm{~S}$ \\
\hline SQDG(36:7) & 835.4666 & 835.4676 & 1.2 & $18: 3-18: 4,20: 4-16: 3$ and $20: 5-16: 2$ & $\mathrm{C}_{45} \mathrm{H}_{71} \mathrm{O}_{12} \mathrm{~S}$ \\
\hline SQDG(34:3-OH) & 831.4928 & 831.4930 & 0.2 & - & $\mathrm{C}_{43} \mathrm{H}_{75} \mathrm{O}_{13} \mathrm{~S}$ \\
\hline SQDG(34:4-OH) & 829.4772 & 829.4752 & -2.4 & - & $\mathrm{C}_{43} \mathrm{H}_{73} \mathrm{O}_{13} \mathrm{~S}$ \\
\hline \multicolumn{6}{|c|}{ SQMG identified as $[\mathrm{M}-\mathrm{H}]^{-}$} \\
\hline SQMG(16:0) & 555.2839 & 555.2838 & -0.2 & $16: 0$ & $\mathrm{C}_{25} \mathrm{H}_{47} \mathrm{O}_{11} \mathrm{~S}$ \\
\hline
\end{tabular}

Table 5. Glycolipids identified in the polar lipidome of Chlorococcum amblystomatis by HILIC-HR-ESI-MS and MS/MS. $C$ carbons, $N$ number of double bonds. ${ }^{*}$ Identified based on the polar head fragment, calculated mass and retention time. ${ }^{* *}$ Identified based on calculated mass and retention time. 


\section{Sulfoquinovosyl diacylglycerol as $[\mathrm{M}-\mathrm{H}]^{-}$}

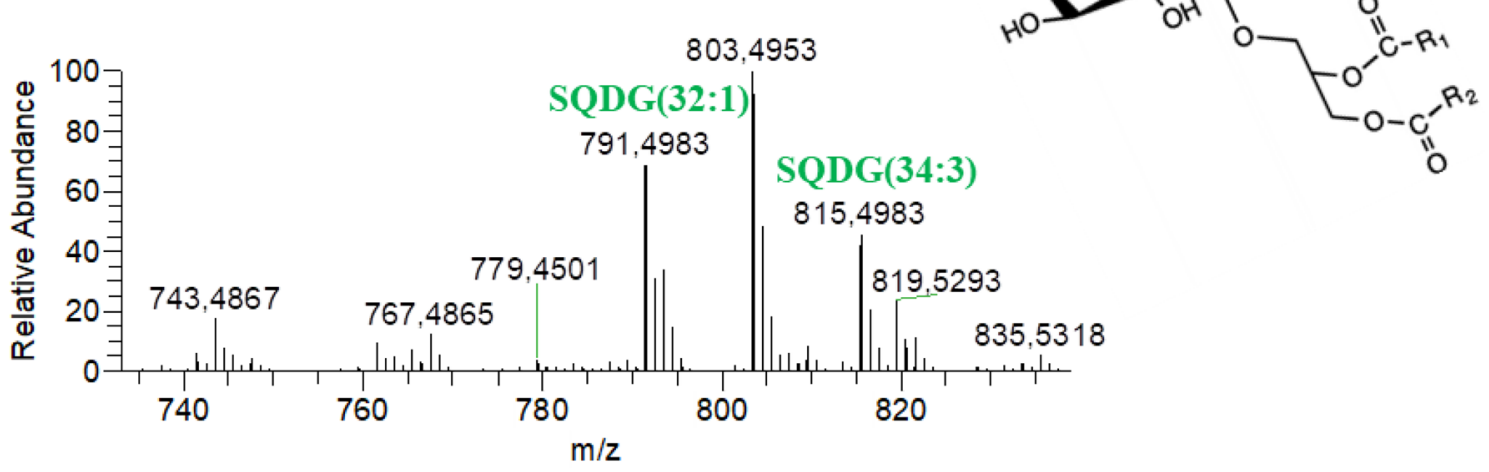

Figure 5. HILIC-HR-ESI-MS spectrum of the classes of glycolipids (GL) identified in negative ion mode as $[\mathrm{M}-\mathrm{H}]^{-}$. This class was sulfoquinovosyl diacylglycerol (SQDG).

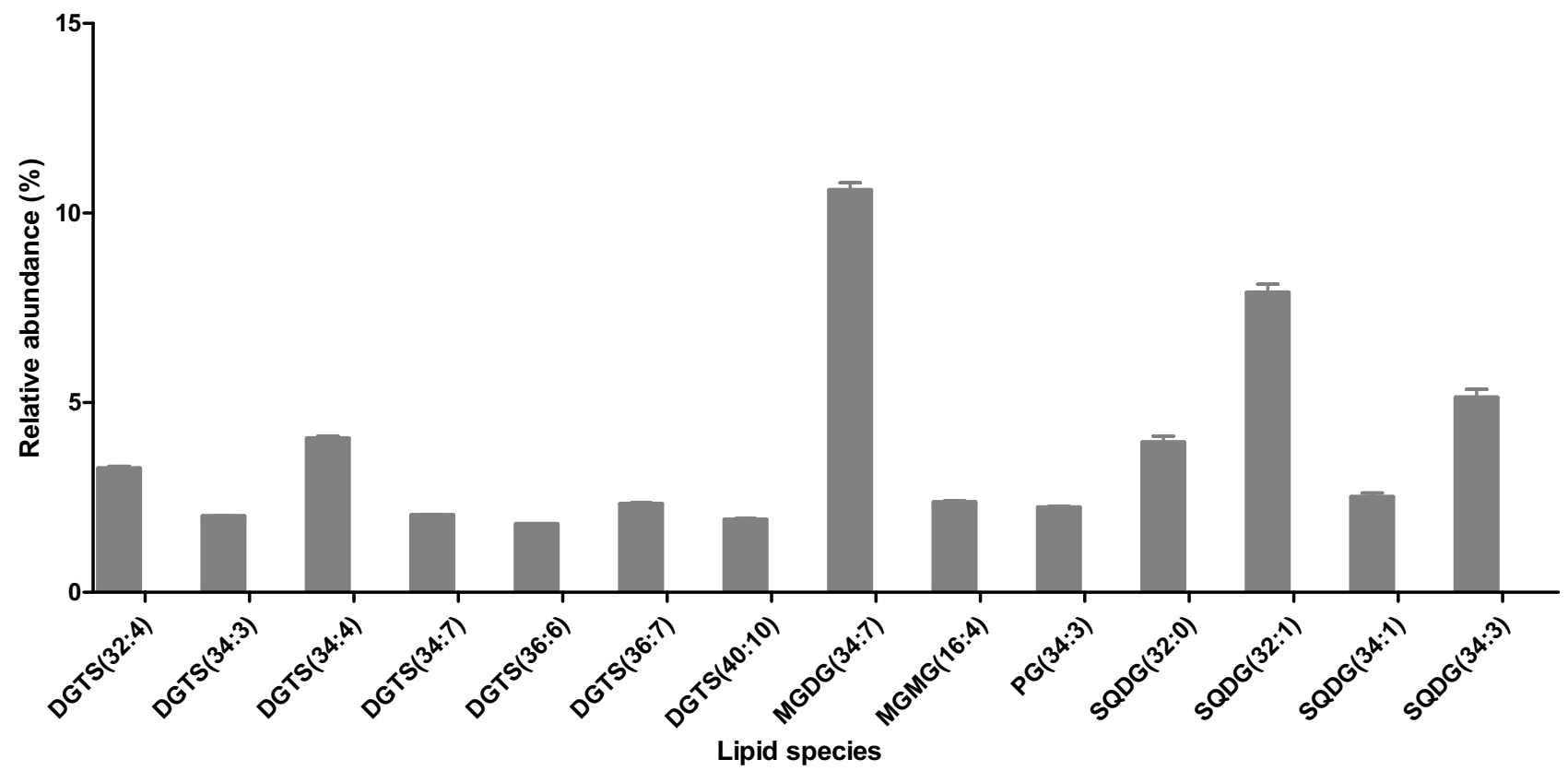

Figure 6. The most abundant lipid species, identified as molecular ions, in the polar lipidome of Chlorococcum amblystomatis, with a relative amount $\geq 1.5 \%$. The results are represented by an average of five samples \pm standard deviations.

and $n-6 / n-3(0.1)$. The AI and TI indexes are commonly used to assess the potential of the matrix to stimulate platelet aggregation, and the lower the indexes the more beneficial it is in reducing the prevalence of heart disease $^{60}$, and have already been used to assess these benefits in microalgae, seaweeds and fish ${ }^{61-65}$. Our results show that these indexes of the lipid extract of Chlorococcum amblystomatis were similar to those observed in fish oils $^{66,67}$. Compared to Spirulina platensis, Nannochloropsis gaditana, Nannochloropsis oculata and Porphyridium tricornutum, Chlorococcum amblystomatis had lower AI values. Their AI values ranged between 0.6 and $1.7^{61}$. As for the TI index, Chlorococcum amblystomatis had lower values than Spirulina platensis, Nannochloropsis gaditana and Porphyridium Cruentum ${ }^{61}$, which were $1.5,3.8$ and 0.6 , respectively. The higher values for $\mathrm{h} / \mathrm{H}$ and the lower $n-6 / n-3$ ratio (1.4 and 0.1 , respectively) reinforce the nutritional potential of the lipid extracts of Chlorococcum amblystomatis.

The profiling of the total lipid extract using high-resolution HILIC-ESI-MS and MS-MS approaches, made it possible, for the first time, to describe the polar lipid composition, in Chlorococcum amblystomatis. The polar lipids identified included glycolipids (GL), phospholipids (PL) and betaine lipids (BL). Data from the literature indicate that algae are a valuable and promising source of polar lipids with bioactive properties, namely, polar lipids esterified to commercially important PUFAs, e.g. eicosapentaenoic acid (EPA). Our profiling made it possible to identify 245 molecular ions, corresponding to a minimum of 350 molecular species: 89 molecular ions of 


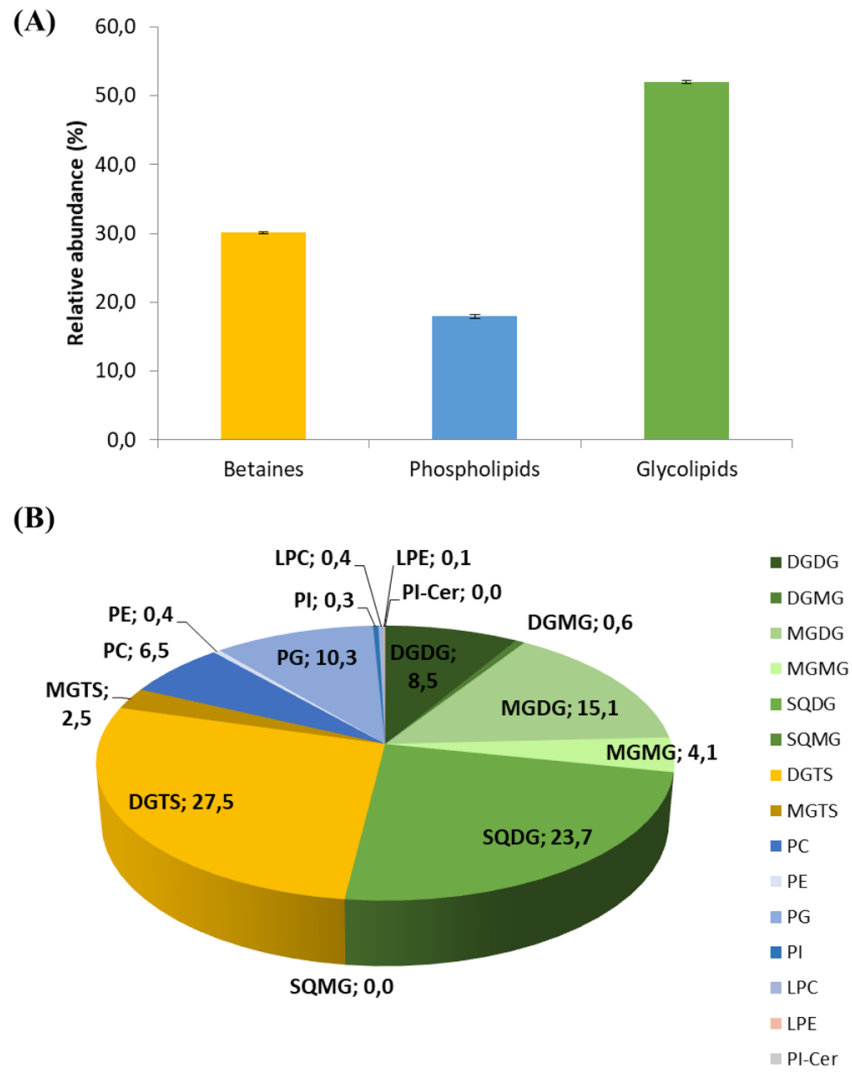

Figure 7. (A) Representation of the relative percentage of betaines, phospholipids and glycolipids present in Chlorococcum amblystomatis. Values are represented by an average of five replicates \pm standard deviation. (B) The relative percentage of each class of lipids.

\section{COX-2}

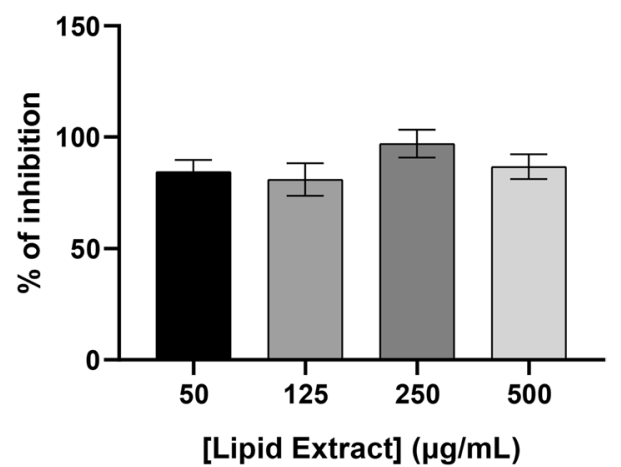

Figure 8. Percent inhibition of $\mathrm{COx}-2$ as a function of the concentration of the lipid extracts of Chlorococcum amblystomatis.

PL, 101 molecular ions of GL (sulfo- and galactolipids), 54 molecular ions of BL and 1 inositol phosphoceramide. These ions were distributed by 15 classes of lipids: PL classes were LPC, PC, LPE, PE, PG and PI; sphingolipid classes were PI-Cer, BL classes were MGTS and DGTS; and GL classes were MGMG, MGDG, DGMG, DGDG, SQDG, and SQDG.

About $45 \%$ of the lipid species detected were esterified to omega-3 fatty acids. Some of these species caught our attention because of their richness in omega-3 PUFAs. The PG (34:3) and the PG (36:5), respectively assigned to PG (16:0-18:3) and PG (16:0-20:5) are two species of PL among the most abundant of Chlorococcum amblystomatis, and both carried omega-3 fatty acids, including EPA. In a previous study in which anti-inflammatory activity was addressed, PG (16:0-20:5) was suggested to promote down-regulated iNOS activity in LPS-stimulated macrophages ${ }^{21,68}$. 
(A) ABTS radical scavenging activity

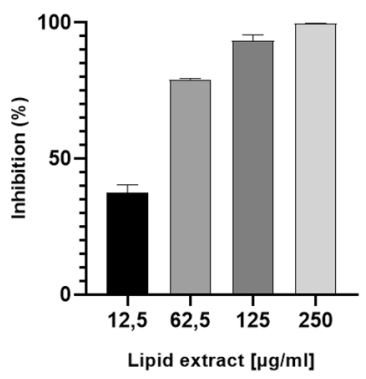

(B) DPPH radical scavenging activity

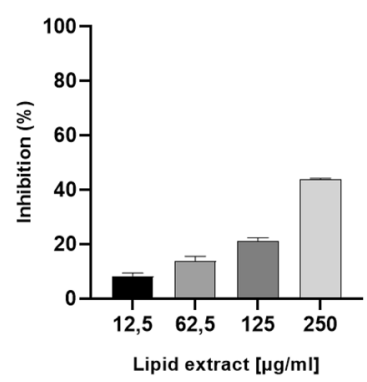

Figure 9. Percent inhibition as a function of the concentration of the lipid extracts of Chlorococcum amblystomatis after 120 min of $\mathrm{ABTS}^{*+}(\mathbf{A})$ and $\mathrm{DPPH}^{\bullet}(\mathbf{B})$ radical scavenging activity.

Since in Chlorococcum amblystomatis there are high amounts of PLs esterified to omega-3 PUFAs and it has been suggested that the PLs are an excellent delivery vehicle for PUFAs ${ }^{18,19}$, this will favour its valorization for food and nutraceutical formulations, where they can be used as value-added ingredients, and also in the cosmetic industry, as they can be used to create moisturizing emulsions ${ }^{69}$. Interestingly, oxidized phosphatidylglycerols (PG) with bonded oxylipins have been identified in this study. Some studies have suggested the anti-inflammatory potential of oxylipins ${ }^{70}$, as reported for Nannochloropsis gaditana and Chlamydomonas debaryana ${ }^{71}$. Indeed, we observed a COX-2 inhibitory activity from the lipid extracts of Chlorcococcum amblystomatis, to which these oxylipins could have contributed. Consequently, extracts rich in polar lipids from Chlorococcum amblystomatis could constitute an interesting opportunity for the nutraceutical or pharmaceutical industries.

The BL identified in the polar lipidome of Chlorococcum amblystomatis, include as the most abundant species, DGTS (34:4), DGTS (32:4), DGTS (36:7), DGTS (34:7), DGTS (34:3), DGTS (40:10) and DGTS (36:6). Moreover, DGTS (40:10) was identified as DGTS (20:5-20:5), a molecular species associated with anti-inflammatory activity, by reducing the production of nitric oxide (NO) ${ }^{72}$. However, BL remains poorly studied to date, and little is known about its bioactive potential.

The polar lipidome of Chlorococcum amblystomatis was particularly rich in glycolipids (MGDG, DGDG and SQDG) which represented approximately half of the polar lipids content (Fig. 7). There is an interest in characterizing GL because of their reported bioactive properties ${ }^{16}$. For example, SQDG of the microalga Porphyridium purpureum have been associated with antioxidant activity, due to their inhibitory effect on the generation of superoxide in peritoneal mononuclear cells ${ }^{73}$. GL with PUFAs have shown anti-inflammatory activity by inhibiting the release of nitric oxide by macrophages ${ }^{20,21}$. There is also a patent for MGDG with EPA to be used as an anti-inflammatory compound ${ }^{74}$. Another interesting application for glycolipids comes from the use of DGDG and SQDG from seaweeds as chemotherapy agents ${ }^{22}$. SQDG esterified in EPA appears to have an antiproliferative effect by inhibiting the key enzymes telomerase ${ }^{23}$ and DNA polymerase- $\alpha$ and $-\beta^{75}$. GL species with known bioactivity, such as MGDG (20:5-16:0), MGDG (20:5-16:1), MGDG (20:5-18:2), MGDG (20:5-20:5), DGDG (20:5-16:0), DGDG (20:5-18:2), DGDG (18:4-16:0) and MGDG (16:4-18:3), were also identified in the Chlorococcum amblystomatis lipidome. These GL species were associated with anti-inflammatory activity by down-regulating the iNOS ${ }^{46,76-78}$.

Regarding sulfur-containing lipids, SQDG (34:3), assigned as SQDG (16:0-18:3), and SQDG (32:0), were among the most abundant SQDG identified in Chlorococcum amblystomatis, and were linked to anti-inflammatory ${ }^{79}$ and antiviral activities ${ }^{80}$, respectively. Also, SQDG (16:0-20:4) and SQDG (16:0-18:4), identified in extracts of Chlorococcum amblystomatis, have been reported for their antiproliferative properties ${ }^{81,82}$. Finally, SQMG (16:0), although detected in low abundance, has been reported to have antimicrobial and antitumor activities ${ }^{83}$.

The polar lipids of Chlorococcum amblystomatis may contribute to the antioxidant activity observed in the DPPH and ABTS radical scavenging assays, as some of the reported polar lipids were previously associated with antioxidant activity ${ }^{73}$. Natural antioxidants are highly sought after for their biological effects ${ }^{84}$ and their wide applications in the food and pharmaceutical sectors ${ }^{85}$. As such, microalgal biomass extracts have been widely explored regarding their potential antioxidant activity ${ }^{86,87}$. Ethanolic and aqueous extracts are mainly composed of phenolic compounds, while methanolic extracts are rich in polar lipids ${ }^{88}$. In a recent study, the DPPH assay was employed to evaluate antioxidant activity on 9 microalgae. The methanolic extracts of the different microalgae strains showed antioxidant activity, with percentage inhibition of DPPH ranging from 15 to $45 \%$ (IC15 to IC45; at $200 \mu \mathrm{g} \mathrm{mL}^{-1}$ extracts concentration) ${ }^{86}$. In this work, the dichloromethane:methanol extracts of Chlorococcum amblystomatis resulted in a percentage of inhibition of $36 \%$ at $200 \mu \mathrm{g} \mathrm{m}^{-1}$, displaying a value better compared to 7 strains of microalgae from the reported work.

COX-2 is an important component of inflammation, associated with pro-inflammatory activity, responsible for the production of prostaglandin $\mathrm{E}_{2}{ }^{89}$. In the present work, the lipid extracts of Chlorococcum amblystomatis exhibit a COX-2 inhibiting activity. It is the first time that such a response has been described in Chlorococcum sp. However, recent work, also measuring COX-2 inhibition, compared the anti-inflammatory potential of aqueous and ethanolic extracts of two Tetraselmis sp. strains and a Skeletonema sp. strain ${ }^{81}$. The highest value of antiinflammatory activity $(82 \pm 2 \%)$ was measured for the ethanolic extract of Skeletonema sp. at a concentration of $1 \mathrm{mg} \mathrm{mL}^{-1}$. Our results showed that the COX inhibition of dichloromethane: methanol extracts of Chlorococcum 
amblystomatis was $87.5 \pm 0.1 \%$, at a concentration of $50 \mu \mathrm{g} \mathrm{mL} \mathrm{m}^{-1}$. These results are consistent with the hypothesis that Chlorococcum amblystomatis has a higher COX-2 inhibitory power than Tetraselmis sp. and Skeletonema sp. The high COX-2 inhibitory activity of Chlorococcum amblystomatis extracts results in promising antiinflammatory potential. Future studies should explore the use of lipid extracts of Chlorococcum amblystomatis in inflammatory cells to explore this anti-inflammatory potential.

\section{Conclusions}

The polar lipidome of Chlorococcum amblystomatis was characterized for the first time in this study. The Chlorococcum amblystomatis strain used revealed a high content of omega-3 PUFAs. PUFAs are associated with several health benefits, such as the prevention of cardiovascular disease. The HILIC-MS/MS lipidomic approach identified 245 molecular ions of polar lipids, in Chlorococcum amblystomatis, revealing to be a microalga rich in glyco- and betaine lipids. Some of the identified polar lipids have already been reported with biological activity, for example, DGTS (20:5-20:5), SQDG (16:0-18:3), MGDG (20:5-18:2) and DGDG (20:5-18:2) which were associated with anti-inflammatory activity. In addition, extracts rich in polar lipids had COX-2 inhibiting activity and antioxidant activity. In conclusion, due to its chemical, biochemical, bioactive, and health-promoting properties, the lipid extracts of Chlorococcum amblystomatis have been found to be of high value for application in food, feed, cosmetic, nutraceutical, and pharmaceutical applications.

\section{Materials and methods}

Reagents. HPLC grade methanol $(\mathrm{MeOH})$, ethanol absolute, and dichloromethane $\left(\mathrm{CH}_{2} \mathrm{Cl}_{2}\right)$, were purchased from Fisher Scientific Ltd. (Loughborough, UK). All other reagents were purchased from major commercial sources. Milli-Q water (Synergy, Millipore Corporation, Billerica, MA, USA) was used. Phospholipid internal standards 1,2-dimyristoyl-sn-glycero-3-phosphocholine (dMPC), 1,2-dimyristoyl-sn-glycero-3-phosphoethanolamine (dMPE), 1,2-dimyristoyl-sn-glycero-3-phospho-(10-rac-glycerol) (dMPG), 1,2-dimyristoyl-sn-glycero-3-phospho-L-serine (dMPS), 1,2-dipalmitoyl-sn-glycero-3-phosphatidylinositol (dPPI), N-palmitoyl-Derythro-sphingosylphosphorylcholine (SM), 1-nonadecanoyl-2-hydroxy-sn-glycero-3-phosphocholine (LPC) were purchased from Avanti Polar Lipids, Inc. (Alabaster, AL), according to the methodology previously described $^{34,35}$. DPPH* was purchased from Aldrich (Milwaukee, WI). 2,20-Azino-bis(3-ethylbenzothiazoline6-sulfonic acid) diammonium salt $\left(\mathrm{ABTS}^{\bullet+}\right.$ ) was obtained from Fluka (Buchs, Switzerland). The 6-hydroxy2,5,7,8-tetramethylchromane-2-carboxylic acid (Trolox) were purchased from Sigma-Aldrich (St Louis, MO, USA). The cyclooxygenase (COX-2) inhibitory screening assay was performed using a commercial kit, Cayman test kit-701080 from Cayman Chemical Company (Ann Arbor, MI, USA). All the other reagents and chemicals used were of the highest grade of purity commercially available.

Microalgae material. The spray-dried biomass of Chlorococcum amblystomatis was supplied by Allmicroalgae, Natural products S.A. located in Pataias, Portugal, Fábrica Cibra Pataias, 2445-287 Pataias. The strain was isolated locally and is deposited in the Allmicroalgae culture collection under the code 0066CA ${ }^{34}$.

Portugal Chlorococcum amblystomatis 0066 CA was cultivated autotrophically in Guillard's F2 culture medium, whose composition was adapted to local water, using nitrates as the source of nitrogen ${ }^{91}$. Briefly, $5 \mathrm{~L}$ flask reactors were cultivated from 7 to 15 days, under continuous exposure to light. Five $5 \mathrm{~L}$ flask reactors were used to inoculate one $0.1 \mathrm{~m}^{3} \mathrm{~L}$ outdoor Flat Panel (FP) reactor, which was later sequentially scaled as follows: 0.25 $\mathrm{m}^{3} \mathrm{~L}$ to a $0.5 \mathrm{~m}^{3} \mathrm{~L}$ to a $1 \mathrm{~m}^{3}$ Flat Panels. Four of the later reactors were used as inoculum of a $10 \mathrm{~m}^{3}$ photobioreactor (PBR). The reactor was operated for 21 days, exposed to the environmental light and temperature conditions, at an average temperature of $15.5^{\circ} \mathrm{C}$ and light irradiance of $20.10 \mathrm{MJ} \mathrm{m}^{-2} \mathrm{day}^{-1}$. $\mathrm{pH}$ was maintained constant, 7.0-8.0, by pulse injections of $\mathrm{CO}_{2}$ and the temperature was kept under $28^{\circ} \mathrm{C}$ by a sprinkler-like irrigation system. After growing period, the biomass was industrially collected by centrifugation and further spray-drying.

Lipid extraction procedure. Lipid extraction was carried out using a mixture of dichloromethane: methanol solvents (DM) (2:1, v/v). The lipids were extracted from $25 \mathrm{mg}$ of lyophilized Chlorococcum amblystomatis biomass using the solvent mixture. The suspension was centrifuged (Selecta JP Mixtasel, Abrera, Barcelona, Spain) at $2000 \mathrm{rpm}$ for $10 \mathrm{~min}$ and the supernatant was collected in a new pre-weighed glass tube. This process was repeated four times until the extraction solvent lost the green colour. The combined supernatants were dried under a stream of nitrogen.

The Folch extraction method was used to the obtained dried supernatants ${ }^{54,92}$. The extracts were redissolved in $2 \mathrm{~mL}$ of dichloromethane, and $1 \mathrm{~mL}$ of methanol and $0.75 \mathrm{~mL}$ of Milli-Q water were added. The mixture was vortexed for $2 \mathrm{~min}$ followed by phase separation by centrifugation at $2000 \mathrm{rpm}$ for $10 \mathrm{~min}$. The organic phase was collected in a new pre-weighed tube, and the aqueous phase was reextracted with $2 \mathrm{~mL}$ of dichloromethane, two more times. The combined organic phases were dried under a stream of nitrogen and weighted.

Each series of extracts was repeated five times and the total lipid content was determined by gravimetry. The yield of lipids extracted from dry biomass extracts (DW) was calculated as follows (Eq. 1):

$$
\text { Lipid content yield }\left(\% \mathrm{DW}, \frac{\mathrm{w}}{\mathrm{w}}\right)=\frac{\text { Weight of the lipid extract }(\mathrm{g})}{\text { Weight of biomass }(\mathrm{g})} \times 100
$$

COX-2 inhibition assay. A commercial cyclooxygenase (COX-2) inhibitory screening assay kit-Cayman test kit-701080 (Cayman Chemical Company, Ann Arbor, MI, USA) - was used to assess their anti-inflammatory potential ${ }^{93}$. This screening assay directly measures the amount of prostaglandin F2 $\alpha$ generated from 
arachidonic acid (AA, C20:4 [n-6]) in the cyclooxygenase reaction. This assay was carried out according to the instructions described by the supplier of the assay kit, using an aliquot of test extract or DMSO. For this assay, lipid extracts of Chlorococcum amblystomatis $(500 \mu \mathrm{g}, 250 \mu \mathrm{g}, 125 \mu \mathrm{g}$ and $50 \mu \mathrm{g})$ were dissolved in DMSO and the final volume of reaction was $1000 \mu$ l. Positive and negative controls were provided by the assay kit protocol. The positive control used inactivated COX-2 enzyme, and negative control used the enzyme with $100 \%$ initial activity without any inhibitor. The assay was performed in three replicates. Interferences were considered by subtracting COX-2 inhibition from the blank assays. The results were expressed as a percentage of inhibited COX-2.

DPPH Radical Scavenging Assay. The antioxidant scavenging activity against the $\alpha, \alpha$-diphenyl- $\beta$ picrylhydrazyl radical ( $\mathrm{DPPH}^{*}$ ) was evaluated as described previously ${ }^{94,95}$ with some modifications. Briefly, 150 $\mu \mathrm{L}$ of an ethanolic dilution of the extracts $\left(25,125,250,500 \mu \mathrm{g} \mathrm{mL}^{-1}\right)$ or $150 \mu \mathrm{L}$ of the Trolox standard solution $\left(5,12.5,25,37.5 \mu \mathrm{mol} \mathrm{L}{ }^{-1}\right.$ in ethanol) were mixed in triplicate with $150 \mu \mathrm{L}$ of a $\mathrm{DPPH}^{\bullet}$ working solution in ethanol (absorbance $\sim 0.9,517 \mathrm{~nm}$ ). The mixture was incubated for $120 \mathrm{~min}$ and the absorbance was measured at $517 \mathrm{~nm}$ every $5 \mathrm{~min}$ (Multiskan GO 1.00.38, Thermo Scientific, Hudson, NH, USA). A control was prepared by replacing the $\mathrm{DPPH}^{*}$ solution with ethanol. The antioxidant activity, expressed as a percentage of inhibition of the DPPH radical, was calculated using the following equation (Eq. 2):

$$
\text { Inhibition } \%=\frac{\left(A b s_{D P P H}-A b s_{\text {Sample }}\right)}{A b s_{D P P H}} \times 100
$$

The activity is expressed in Trolox Equivalents, which were calculated using Eq. 2, where IC40 values are the concentration of sample or of Trolox that induces the reduction the DPPH• radical to 40\%) (Eq. 3):

$$
\mathrm{TE}=\frac{\mathrm{IC} 40 \text { Trolox }(\mu \mathrm{mol} / \mathrm{g})}{\text { IC40 of samples }(\mu \mathrm{g} / \mathrm{mL})} \times 1000 .
$$

ABTS radical cation scavenging assay. The antioxidant scavenging activity against the 2,20-azinobis-3-ethylbenzothiazoline-6-sulfonic acid radical cation $\left(\mathrm{ABTS}^{\bullet+}\right)$ was evaluated using a method previously described ${ }^{94,96}$ with some modifications. Briefly, $150 \mu \mathrm{L}$ of an ethanolic dilution of the extracts $(25,125,250$, $\left.500 \mu \mathrm{g} \mathrm{mL}^{-1}\right)$ or $150 \mu \mathrm{L}$ of the Trolox standard solution $\left(5,12.5,25,37.5 \mu \mathrm{mol} \mathrm{L}^{-1}\right.$ in ethanol) were mixed in triplicate with $150 \mu \mathrm{L}$ of an $\mathrm{ABTS}^{\bullet+}$ working solution in ethanol (absorbance $\sim 0.9,734 \mathrm{~nm}$ ). The mixture was incubated for $120 \mathrm{~min}$ and the absorbance was measured at $734 \mathrm{~nm}$ every $5 \mathrm{~min}$ (Multiskan GO 1.00.38, Thermo Scientific, Hudson, NH, USA). A control was prepared by replacing the ABTS ${ }^{++}$solution with ethanol. The antioxidant activity, expressed as a percentage of inhibition of the ABTS radical (IC40 substituted by IC50), was calculated using Eq. (2) $\left(\mathrm{Abs}_{\mathrm{DPPH}}{ }^{\circ}\right.$ substituted by $\left.\mathrm{Abs}_{\mathrm{ABTS}}{ }^{\cdot+}\right)$ and expressed in Trolox Equivalents (Eq. 3).

Analysis of fatty acids by gas chromatography-mass spectrometry (GC-MS). The fatty acid methyl esters (FAMEs) were prepared from total lipid extracts of Chlorococcum amblystomatis by transmethylation reaction using a methanolic solution of potassium hydroxide $(2.0 \mathrm{M})$ according to the methodology previously described ${ }^{90}$. A volume of $2.0 \mu \mathrm{L}$ of a hexane solution containing FAMEs and $1.03 \mu \mathrm{g} \mathrm{mL}^{-1}$ of methyl nonadecanoate (Sigma, St. Louis, MO, USA) as internal standard was injected in a chromatography-mass spectrometry (GC-MS) (Agilent Technologies 8860 GC System, Santa Clara, CA, USA) equipped with a DB-FFAP column with the following specifications: $30 \mathrm{~m}$ long, $0.32 \mathrm{~mm}$ internal diameter, and $0.25 \mu \mathrm{m}$ film thickness (J \& W Scientific, Folsom, CA, USA). The GC equipment was connected to an Agilent 5977B Mass Selective Detector operating with electron impact ionization at $70 \mathrm{eV}$ and a scanning range of $\mathrm{m} / z 50-550$ ( $1 \mathrm{~s}$ cycle in a full scan mode). The following conditions were used: helium as carrier gas (constant flow $1.4 \mathrm{~mL} \mathrm{~min}{ }^{-1}$ ), inlet temperature $220^{\circ} \mathrm{C}$, detector temperature $230^{\circ} \mathrm{C}$, injection volume $2 \mu \mathrm{L}$ (splitless). The oven temperature was

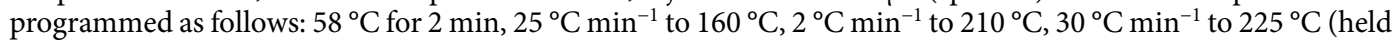
for $20 \mathrm{~min}$ ). The data acquisition software used was GCMS5977B/Enhanced MassHunter. Data were analysed using Agilent MassHunter Qualitative Analysis 10.0 software. The identification of FA was carried out by comparison of the MS spectrum with the NIST chemical database library and confirmed with the literature reports. Five independent replicates were injected.

Atherogenic, thrombogenic and hypocholesterolemic/hypercholesterolemic indices. The atherogenic, thrombogenic and hypocholesterolemic/hypercholesterolemic indices $(\mathrm{h} / \mathrm{H})$ were calculated as the content ratio of saturated fatty acids (SFA)/unsaturated FA, as monounsaturated fatty acids (MUFAs) and omega-3 and omega-6 PUFAs, using the following formula (Eqs. 4, 5 and 6), as proposed by Ulbricht and Southgate ${ }^{97}$

$$
\begin{aligned}
& \text { Atherogenic index }(\mathrm{AI})=\frac{[\mathrm{C} 12: 0+4 *(\mathrm{C} 14: 0)+\mathrm{C} 16: 0]}{\left[\sum \operatorname{MUFA}+\sum(\mathrm{n}-6)+\sum(\mathrm{n}-3)\right]} \\
& \text { Thrombogenic index }(\mathrm{TI})=\frac{[\mathrm{C} 14: 0+\mathrm{C} 16: 0+\mathrm{C} 18: 0]}{\left[0.5^{\star} \sum \text { MUFA }+0.5^{\star} \sum(\mathrm{n}-6)+3 * \sum(\mathrm{n}-3)+\left(\frac{\sum(\mathrm{n}-6)}{\sum(\mathrm{n}-3)}\right)\right]}
\end{aligned}
$$




$$
\text { hypocholesterolemic/Hypercholesterolemic ratio }(\mathrm{h} / \mathrm{H})=\frac{[\mathrm{C} 18: 1 \omega 9+18: 2 \omega 6+18: 3 \omega 3+\mathrm{C} 20: 4 \omega 6+\mathrm{C} 20: 5 \omega 3]}{[\mathrm{C} 14: 0+\mathrm{C} 16: 0]}
$$

Polar lipidome analysis by hydrophilic interaction liquid chromatography coupled to high-resolution tandem mass spectrometry (HILIC-HR-MS/MS). The polar lipidome was determined according to the methodology previously described ${ }^{34,35}$. The dried samples were dissolved in $\mathrm{CH}_{2} \mathrm{Cl}_{2}$ to a final concentration of $1 \mathrm{ug} \mathrm{uL}^{-1}$. From each sample, a volume of $10 \mu \mathrm{L}(10 \mu \mathrm{g}$ of lipid extract) was taken and transferred to an appropriate vial, followed by the addition of $86 \mu \mathrm{L}$ of a solvent system composed of two mobile phases in a proportion of $90 \% \mathrm{v} / \mathrm{v}$ eluent $\mathrm{B}$ and $10 \% \mathrm{v} / \mathrm{v}$ eluent $\mathrm{A}$ and $4 \mu \mathrm{L}$ of a mixture of internal standards (dMPC $-0.02 \mu \mathrm{g}, \mathrm{dMPE}-0.02 \mu \mathrm{g}, \mathrm{SM}(17: 0 / \mathrm{d} 18: 1)-0.02 \mu \mathrm{g}, \mathrm{LPC}-0.02 \mu \mathrm{g}, \mathrm{dPPI}-0.08 \mu \mathrm{g}, \mathrm{dMPG}-0.012 \mu \mathrm{g}$, $\mathrm{dMPS}-0.04 \mu \mathrm{g}$ ). The composition of eluent B was $60 \% \mathrm{v} / \mathrm{v}$ of acetronitrile, $40 \% \mathrm{v} / \mathrm{v}$ of methanol, and $5 \mathrm{mM}$ of ammonium acetate and the composition of eluent A was $50 \% \mathrm{v} / \mathrm{v}$ of acetonitrile, $25 \% \mathrm{v} / \mathrm{v}$ of methanol, $25 \% \mathrm{v} / \mathrm{v}$ of water, and $5 \mathrm{mM}$ ammonium acetate.

The lipids were separated by hydrophilic interaction liquid chromatography (HILIC) using a microbore Ascentis Si column $(10 \mathrm{~cm} \times 1.0 \mathrm{~mm}, 3 \mu \mathrm{m}$; Sigma-Aldrich) and a high performance-liquid chromatography (HPLC) system (Ultimate 3000 Dionex, Thermo Fisher Scientific, Bremen, Germany) with an autosampler coupled to the Q-Exactive hybrid quadrupole Orbitrap mass spectrometer (Thermo Fisher Scientific, Bremen, Germany). A $5 \mu \mathrm{L}$ aliquot of each sample mixture was injected into the HPLC column, at a flow rate of $50 \mu \mathrm{L}$ $\mathrm{min}^{-1}$ and a temperature of $35^{\circ} \mathrm{C}$. The following gradient was applied: $10 \% \mathrm{~A}(0-2 \mathrm{~min}), 10-90 \% \mathrm{~A}(2-15 \mathrm{~min})$, $90 \%$ A (15-17 min). The mass spectrometer with Orbitrap technology was operated simultaneously in positive (electrospray voltage $3.0 \mathrm{kV}$ ) and negative (electrospray voltage $-2.7 \mathrm{kV}$ ) modes, with the following configuration: high resolution with 70,000, AGC target of $1 \times 10^{6}$, capillary temperature $250{ }^{\circ} \mathrm{C}$, and sheath gas flow of $15 \mathrm{U}$. The tandem mass spectrometry experiments were performed according to the following configuration: resolution of 17,500, AGC target of $1 \times 10^{5}$, with one full scan mass spectrum and 10 data-dependent MS/MS scans. The cycles were repeated continuously throughout the experiments with the dynamic exclusion of $60 \mathrm{~s}$ and an intensity threshold of $1 \times 10^{4}$. Normalized collision energy (CE) ranged between 25 , 30, and $35 \mathrm{eV}$. Data acquisition was performed using the Xcalibur data system (V3.3, Thermo Fisher Scientific, USA). Five independent biological replicas were carried out.

Data analysis. To identify the classes of the polar lipids in the lipid extracts acquired spectra were analysed using Xcalibur v3.3 (Thermo Fisher Scientific, USA). The identification was carried out according to a standard approach in our laboratory ${ }^{98}$ : this approach consists of the localization of the species according to the retention time of internal standards (information related to internal standards can be found in Supplementary Table S1), accurate mass measurements $(5 \mathrm{ppm})$ and identification of recurring fragmentation patterns (Supplementary Figures S1-S13) and their comparison with those of internal standards and published information on fragmentation patterns ${ }^{98-101}$. After identification, the quantification of molecular species was carried out by integrating the chromatographic peaks using the MZmine v2.42 software. The software allows filtering and smoothing, peak detection, peak processing and assignment against an internal database ${ }^{102}$. All peaks of raw intensity bellow $1 \times 10^{4}$ were excluded.

Relative quantification was performed by exporting the values of the peak areas to a computer spreadsheet (Excel, Microsoft, Redmond, WA). To normalize the data, the peak areas of the extracted ion chromatograms (EIC) of each lipid molecular species were divided by the EIC peak areas of the selected internal standards. Relative abundances were calculated using dMPC, dMPE, SM(17:0/d18:1), LPC, dPPI, dMPG and dMPS as internal standards. To normalize DGTS and MGTS we used PE as internal standard, for SQDG we used PG as internal standard, and for MGDG, DGDG, DGMG and MGMG, we used Ceramide as internal standard. For clear visualization, normalized data was transformed in percentage. The normalized data was calculated as follows (Eq. 7):

$$
\text { Relative quantification } \%=\frac{\frac{(\text { EIC lipid species })}{\text { (EIC internal standard })}}{\sum \text { Total Normalized EICs }} \times 100
$$

The relative percentage of betaines, phospholipids and glycolipids was calculated as follows (Eq. 8):

$$
\text { Relative quantification classes } \%=\frac{\sum \text { Normalized EICs each class }}{\sum \text { Total Normalized EICs }} \times 100
$$

The relative percentage of the distribution of omega- 3 and omega- 6 fatty acids across the different main classes of polar lipids (betaines, phospholipids and glycolipids) was calculated as follows (Eq. 9):

Relative quantification of $\omega-3 / \omega-6$ polar lipids $=\sum$ Relative percentage of each species calculated in Equation 7

Raw abundances, normalized data and relative quantification can be found on the additional spreadsheet (Supplementary file S1).

Data availability

Raw datasets generated during this study are available from the corresponding authors upon reasonable request. 
Received: 28 October 2020; Accepted: 3 February 2021

Published online: 23 February 2021

\section{References}

1. Zhu, L. D., Li, Z. H. \& Hiltunen, E. Strategies for lipid production improvement in microalgae as a biodiesel feedstock. Biomed. Res. Int. 2016, 1-8 (2016).

2. Nations U. Transforming Our World: United Nations the 2030 Agenda for Sustainable Development. In: United Nations Sustainable Development Summit. 2015.

3. Khan, M. I., Shin, J. H. \& Kim, J. D. The promising future of microalgae: current status, challenges, and optimization of a sustainable and renewable industry for biofuels, feed, and other products. Microb. Cell Fact. 17(1), 1-21 (2018).

4. Ravindran, B. et al. Microalgae potential and multiple roles-current progress and future prospects-an overview. Sustainability. 8(12), 1-16 (2016).

5. Hamed, I. The evolution and versatility of microalgal biotechnology: A review. Compr. Rev. Food Sci. Food Saf. 15(6), 1104-1123 (2016).

6. Koller, M., Muhr, A. \& Braunegg, G. Microalgae as versatile cellular factories for valued products. Algal Res. 6, 52-63 (2014).

7. Afzal, I., Shahid, A., Ibrahim, M., Liu, T., Nawaz, M., \& Mehmood, M.A. Microalgae: A promising feedstock for energy and high-value products. Algae based polymers, blends, and composites: chemistry, biotechnology and materials science. Elsevier, pp. 55-75 (2017).

8. Mata, T. M., Martins, A. A. \& Caetano, N. S. Microalgae for biodiesel production and other applications: A review. Renew. Sustain. Energy Rev. 14(1), 217-232 (2010).

9. Pulz, O. \& Gross, W. Valuable products from biotechnology of microalgae. Appl. Microbiol. Biotechnol. 65, 635-648 (2004).

10. Patil, V., Tran, K. Q. \& Giselrød, H. R. Towards sustainable production of biofuels from microalgae. Int. J. Mol. Sci. 9(7), 1188-1195 (2008).

11. Ganesan, V. S. H. Biomass from microalgae: an overview. Oceanogr. Open Access. 2(1), 1-7 (2014).

12. Acién Fernández, F. G., Gómez-Serrano, C. \& Fernández-Sevilla, J. M. Recovery of nutrients from wastewaters using microalgae. Front. Sustain. Food Syst. 2(59), 1-13 (2018).

13. Kay, R. A. \& Barton, L. L. Microalgae as Food and Supplement. Crit. Rev. Food Sci. Nutr. 30(6), 555-573 (1991).

14. da Vaz, B. \& S, Moreira JB, Morais MG de, Costa JAV, ,. Microalgae as a new source of bioactive compounds in food supplements. Curr. Opin. Food Sci. 7, 73-77 (2016).

15. Ahmmed, M. K., Ahmmed, F., Tian, H., Carne, A. \& Bekhit, A. E. D. Marine omega-3 (n-3) phospholipids: A comprehensive review of their properties, sources, bioavailability, and relation to brain health. Compr. Rev. Food. Sci. Food Saf. 19(1), 64-123 (2020).

16. da Costa, E., Silva, J., Mendonça, S. H., Abreu, M. H. \& Domingues, M. R. Lipidomic approaches towards deciphering glycolipids from microalgae as a reservoir of bioactive lipids. Mar. Drugs. 14(5), 1 (2016).

17. Schneider, H., Braun, A., Füllekrug, J., Stremmel, W. \& Ehehalt, R. Lipid based therapy for ulcerative colitis-modulation of intestinal mucus membrane phospholipids as a tool to influence inflammation. Int. J. Mol. Sci. 11(10), 4149-4164 (2010).

18. Lagarde, M. et al. Lysophosphatidylcholine as a carrier of docosahexaenoic acid to target tissues. World Rev. Nutr. Diet. 88, 173-177 (2001).

19. Picq, M. et al. DHA metabolism: Targeting the brain and lipoxygenation. Mol. Neurobiol. 42(1), 48-51 (2010).

20. Lopes, G., Daletos, G., Proksch, P., Andrade, P. B. \& Valentão, P. Anti-inflammatory potential of monogalactosyl diacylglycerols and a monoacylglycerol from the edible brown seaweed Fucus spiralis linnaeus. Mar Drugs. 12(3), 1406-1418 (2014).

21. Banskota, A. H. et al. Polar lipids from the marine macroalga Palmaria palmata inhibit lipopolysaccharide-induced nitric oxide production in RAW264.7 macrophage cells. Phytochemistry 101, 101-108 (2014).

22. Hossain, Z., Kurihara, H., Hosokawa, M. \& Takahashi, K. Growth inhibition and induction of differentiation and apoptosis mediated by sodium butyrate in Caco-2 cells with algal glycolipids. Vitr Cell Dev. Biol. Anim. 41(5-6), 154-159 (2005).

23. Eitsuka, T., Nakagawa, K., Igarashi, M. \& Miyazawa, T. Telomerase inhibition by sulfoquinovosyldiacylglycerol from edible purple laver (Porphyra yezoensis). Cancer Lett. 212(1), 15-20 (2004).

24. Yao, L., Gerde, J. A., Lee, S. L., Wang, T. \& Harrata, K. A. Microalgae lipid characterization. J Agric Food Chem. 63(6), 1773-1787 (2015).

25. Li, S. et al. The major lipid changes of some important diet microalgae during the entire growth phase. Aquaculture 428-429, 104-110 (2014).

26. Meng, Y., Cao, X., Yao, C., Xue, S. \& Yang, Q. Identification of the role of polar glycerolipids in lipid metabolism and their acyl attribution for TAG accumulation in Nannochloropsis oceanica. Algal Res. 24, 122-129 (2017).

27. Liu, P., Corilo, Y. E. \& Marshall, A. G. Polar lipid composition of biodiesel algae candidates nannochloropsis oculata and haematococcus pluvialis from nano liquid chromatography coupled with negative electrospray ionization $14.5 \mathrm{~T}$ fourier transform ion cyclotron resonance mass spe. Energy Fuels 30(10), 8270-8276 (2016).

28. Jouhet, J. et al. LC-MS/MS versus TLC plus GC methods: Consistency of glycerolipid and fatty acid profiles in microalgae and higher plant cells and effect of a nitrogen starvation. PLoS ONE 12(8), e0182423 (2017).

29. Hasan, C. M. M., Aftabuddin, S., Sharif, M. \& Khan, M. Triacylglycerol profile of a microalga Chlorococcum sp. as a potential biofuel feedstock. J. Bangladesh Acad. Sci. 40(2), 147-153 (2016).

30. Lv, J. et al. The performance of a self-flocculating microalga Chlorococcum sp GD in wastewater with different ammonia concentrations. Int. J. Environ. Res. Public Health. 15(3), 434 (2018)

31. Ambati, R. R. et al. Industrial potential of carotenoid pigments from microalgae: Current trends and future prospects. Crit. Rev. Food Sci. Nutr. 59(12), 1880-1902 (2019).

32. Ghosh, A. et al. Biochemical characterization of microalgae collected from north east region of India advancing towards the algae-based commercial production. Asia Pacific J. Chem. Eng. 12(5), 745-754 (2017).

33. Sassi, K. K. B., da Silva, J. A., Calixto, C. D., Sassi, R. \& da Sassi, C. F. Metabolites of interest for food technology produced by microalgae from the Northeast Brazil. Rev Ciência Agronômica 50(1), 54-65 (2019).

34. Correia, N. et al. Isolation, identification and biotechnological applications of a novel, robust, free-living Chlorococcum (Oophila) amblystomatis strain isolated from a local pond. Appl. Sci. 10(9), 3040-3054 (2020).

35. Weiss, A., Johannisbauer, W., Gutsche, B., Cordero, B., Martin, L., Rodriguez, H., \& Vargas, M.A. Obraztsova I. Process for obtaining lutein from algae. US; p. U.S. Patent No. 20070196893A1 12 january 2006.

36. Idrissi Abdelkhalek, E. A., Mohamed, B., Mohammed, A., \& Lotfi, A. Growth performance and biochemical composition of nineteen microalgae collected from different Moroccan reservoirs. Mediterr Mar. Sci. 17(1):323 (2016).

37. Liu, B.H., \& Lee, Y.K. Secondary carotenoids formation by the green alga Chlorococcum sp. J. Appl. Phycol. 1, 301-307 (2000).

38. Prabakaran P, Tech DR-CJ. Selection of microalgae for accumulation of lipid production under different growth conditions. Chemistry (Easton) 1:131-7 (2013).

39. Mahapatra, D. M. \& Ramachandra, T. V. Algal biofuel: Bountiful lipid from chlorococcum sp. proliferating in municipal wastewater. Curr. Sci. 105(1), 47-55 (2013). 
40. Harwati, T. U., Willke, T. \& Vorlop, K. D. Characterization of the lipid accumulation in a tropical freshwater microalgae Chlorococcum sp. Bioresour. Technol. 121, 54-60 (2012).

41. Yang, M. et al. Triacylglycerol accumulates exclusively outside the chloroplast in short-term nitrogen-deprived Chlamydomonas reinhardtii. Biochim. Biophys. Acta Mol. Cell Biol. Lipids. 1863(12), 1478-1487 (2018).

42. White, D. et al. Modulation of polar lipid profiles in Chlorella sp. response to nutrient limitation. Metabolites. 9(3), 39 (2019).

43. Řezanka, T. \& Podojil, M. Preparative separation of algal polar lipids and of individual molecular species by high-performance liquid chromatography and their identification by gas chromatography-mass spectrometry. J. Chromatogr. A. 463, 397-408 (1989).

44. da Costa, E. et al. Decoding bioactive polar lipid profile of the macroalgae Codium tomentosum from a sustainable IMTA system using a lipidomic approach. Algal Res. 12, 388-397 (2015).

45. Melo, T. et al. Lipidomics as a new approach for the bioprospecting of marine macroalgae: Unraveling the polar lipid and fatty acid composition of chondrus crispus. Algal Res. 8, 181-191 (2015).

46. da Costa, E. et al. Lipidomic Signatures Reveal Seasonal Shifts on the Relative Abundance of High-Valued Lipids from the Brown Algae Fucus vesiculosus. Mar Drugs. 17(6), 335 (2019).

47. Lopes, D. et al. Lipidomic signature of the green macroalgae Ulva rigida farmed in a sustainable integrated multi-trophic aquaculture. J. Appl. Phycol. 1, 1-13 (2018).

48. Rey F, Lopes D, Maciel E, Monteiro J, Skjermo J, Funderud J, Raposo D, Domingues P, Calado R, Domingues MR. Polar lipid profile of Saccharina latissima, a functional food from the sea. Algal Res. 39, 101473 (2019).

49. Couto, D. et al. Glycosphingolipids and oxidative stress: Evaluation of hydroxyl radical oxidation of galactosyl and lactosylceramides using mass spectrometry. Chem. Phys. Lipids. 191, 106-114 (2015).

50. Couto, D. et al. New insights on non-enzymatic oxidation of ganglioside GM1 using mass spectrometry. J. Am. Soc. Mass Spectrom. 27(12), 1 (2016).

51. Han, X. \& Gross, R. W. Electrospray ionization mass spectroscopic analysis of human erythrocyte plasma membrane phospholipids. Proc. Natl. Acad. Sci. USA. 91(22), 10635-10639 (1994).

52. Hermansson, M., Uphoff, A., Käkelä, R. \& Somerharju, P. Automated quantitative analysis of complex lipidomes by liquid chromatography/mass spectrometry. Anal. Chem. 77(7), 2166-2175 (2005).

53. Ejsing, C. S. et al. Automated identification and quantification of glycerophospholipid molecular species by multiple precursor ion scanning. Anal. Chem. 78(17), 6202-6214 (2006).

54. Figueiredo, A. R. P., da Costa, E., Silva, J., Domingues, M. R. \& Domingues, P. The effects of different extraction methods of lipids from Nannochloropsis oceanica on the contents of omega-3 fatty acids. Algal Res. 41(12), 1 (2019).

55. Olaizola, M., Grewe, C. Commercial microalgal cultivation systems. In: Grand challenges in algae biotechnology. p. 3-34 (2019).

56. Do, N. H., Ramli, A. \& Kee, L. M. A review on methods used in analysis of microalgae lipid composition. J. Japan Inst. Energy 96(12), 532-537 (2017).

57. Mankad, D. et al. A randomized, placebo controlled trial of omega-3 fatty acids in the treatment of young children with autism. Mol. Autism. 6(1), 1-11 (2015).

58. Barros, R. et al. Dietary intake of $\alpha$-linolenic acid and low ratio of n-6:n-3 PUFA are associated with decreased exhaled NO and improved asthma control. Br. J. Nutr. 106(3), 441-450 (2011).

59. Rennie, K. L., Hughes, J., Lang, R. \& Jebb, S. A. Nutritional management of rheumatoid arthritis: A review of the evidence. J. Hum. Nutr. Diet. 16(2), 97-109 (2003).

60. Šimat, V., Bogdanović, T., Poljak, V. \& Petričević, S. Changes in fatty acid composition, atherogenic and thrombogenic health lipid indices and lipid stability of bogue (Boops boops Linnaeus, 1758) during storage on ice: Effect of fish farming activities. J. Food Compos. Anal. 40, 120-125 (2015).

61. Matos, Â. P. et al. Chemical characterization of six microalgae with potential utility for food application. J. Am. Oil Chem. Soc. 93(7), 963-972 (2016)

62. Marques, B., Lillebø, A. I. \& Domingues, M. Effect of high-pressure processing (HPP) on the fatty acid profile of different sized Ragworms (Hediste diversicolor) cultured in an integrated multi-trophic aquaculture (IMTA) system. Molecules 24(24), 1-11 (2019).

63. Lopes, D., Melo, T., Rey, F., Meneses, J., Monteira, F.L., Helguero, L.A., Abreu, M.H., Lillebø, A.I., Calado, R., \& Domingues, M.R. Valuing bioactive lipids from green, red and brown macroalgae from aquaculture, to foster functionality and biotechnological applications. Molecules (2020).

64. Łuczyńska, J., Paszczyk, B., Nowosad, J. \& Łuczyński, M. J. Mercury, fatty acids content and lipid quality indexes in muscles of freshwater and marine fish on the polish market: Risk assessment of fish consumption. Int. J. Environ. Res. Public Health 14(10), 1-17 (2017).

65. Magdugo, R. P. et al. An analysis of the nutritional and health values of Caulerpa racemosa (Forsskål) and Ulva fasciata (Delile)Two chlorophyta collected from the Philippines. Molecules 25(12), 1-23 (2020).

66. Rueda, F. M. et al. Differences in tissue fatty acid composition between reared and wild sharpsnout sea bream, Diplodus puntazzo (Cetti, 1777). Br. J. Nutr. 86(5), 617-622 (2001).

67. Valfré, F., Caprino, F. \& Turchini, G. M. The health benefit of seafood. Vet. Res. Commun. 27(1), 507-512 (2003).

68. Maciel, E. et al. Bioprospecting of marine macrophytes using MS-based lipidomics as a new approach. Mar. Drugs. 14(3), 1 (2016).

69. Burri, L., Hoem, N., Banni, S. \& Berge, K. Marine Omega-3 phospholipids: Metabolism and biological activities. Int. J. Mol. Sci. 13(11), 15401-15419 (2012).

70. Gabbs, M., Leng, S., Devassy, J. G. \& Aukema, H. M. Advances in our understanding of oxylipins. Am. Soc. Nutr. 6, 513-540 (2015).

71. De Los, R. C. et al. Oxylipins from the microalgae Chlamydomonas debaryana and Nannochloropsis gaditana and their activity as TNF- $\alpha$ inhibitors. Phytochemistry 102, 152-161 (2014).

72. Banskota, A. H., Stefanova, R., Sperker, S. \& McGinn, P. J. New diacylglyceryltrimethylhomoserines from the marine microalga Nannochloropsis granulata and their nitric oxide inhibitory activity. J. Appl. Phycol. 25(5), 1513-1521 (2013).

73. Bergé, J. P., Debiton, E., Dumay, J., Durand, P. \& Barthomeuf, C. In vitro anti-inflammatory and anti-proliferative activity of sulfolipids from the red alga Porphyridium cruentum. J. Agric. Food Chem. 50(21), 6227-6232 (2002).

74. Winget, R.R. Anti-inflammatory compositions containing monogalactosyl dieicosapentaenoyl glycerol and methods relating thereto. United States of America; 5,767,095.

75. Ohta, K. et al. Action of a new mammalian DNA polymerase inhibitor, sulfoquinovosyldiacylglycerol. Biol. Pharm. Bull. 22(2), $111-116(1999)$

76. Banskota, A. H., Stefanova, R., Gallant, P. \& McGinn, P. J. Mono- and digalactosyldiacylglycerols: Potent nitric oxide inhibitors from the marine microalga Nannochloropsis granulata. J. Appl. Phycol. 25(2), 349-357 (2013).

77. Banskota, A. H., Gallant, P., Stefanova, R., Melanson, R. \& O'Leary, S. J. B. Monogalactosyldiacylglycerols, potent nitric oxide inhibitors from the marine microalga Tetraselmis chui. Nat. Prod. Res. 27(12), 1084-1090 (2013).

78. Banskota, A. H. et al. Five new galactolipids from the freshwater microalga Porphyridium aerugineum and their nitric oxide inhibitory activity. J. Appl. Phycol. 25(4), 951-960 (2013). 
79. Bruno, A. et al. Selective in vivo anti-inflammatory action of the galactolipid monogalactosyldiacylglycerol. Eur. J. Pharmacol. 524(1-3), 159-168 (2005).

80. Wang, H. et al. Antiviral activity of a sulfoquinovosyldiacylglycerol (SQDG) compound isolated from the green alga Caulerpa racemosa. Bot. Mar. 50(3), 185-190 (2007).

81. Maeda, N. et al. Anti-tumor effects of the glycolipids fraction from spinach which inhibited DNA polymerase activity. Nutr. Cancer. 57(2), 216-223 (2007).

82. Tsai, C. J. \& Sun, P. B. Identification of sulfoglycolipid bioactivities and characteristic fatty acids of marine macroalgae. J. Agric. Food Chem. 60(34), 8404-8410 (2012).

83. El Baz, F. K., El Baroty, G. S., Abd El Baky, H. H., Abd El-Salam, O. I. \& Ibrahim, E. A. Structural characterization and biological activity of sulfolipids from selected marine algae. Grasas Aceites 64(5), 561-571 (2013).

84. Xu, D.-P. et al. Natural antioxidants in foods and medicinal plants: Extraction, assessment and resources. Int. J. Mol. Sci. 18(1), 1 (2017).

85. Antioxidant, M. S. \& Applications, I. Res. Rev. J. Pharmacol. Toxicol. Stud. 4(4), 1-10 (2016).

86. Banskota, A. H., Sperker, S., Stefanova, R., McGinn, P. J. \& O'Leary, S. J. B. Antioxidant properties and lipid composition of selected microalgae. J. Appl. Phycol. 31(1), 309-318 (2019).

87. Cardoso, C. et al. Lipid composition and some bioactivities of 3 newly isolated microalgae (Tetraselmis sp. IMP3, Tetraselmis sp. CTP4, and Skeletonema sp.). Aquac Int. 28(2), 711-727 (2020).

88. Esmaeili, A. \& Khakpoor, M. Biological activities and chemical composition of solvent extracts of Stoechospermum marginatum (C. Agardh). Acta Biochim. Pol. 59(4), 581-585 (2012).

89. Ricciotti, E. \& Fitzgerald, G. A. Prostaglandins and inflammation. Arterioscler. Thromb. Vasc. Biol. 31(5), 986-1000 (2011).

90. Aued-Pimentel, S., Lago, J. H. G., Chaves, M. H. \& Kumagai, E. E. Evaluation of a methylation procedure to determine cyclopropenoids fatty acids from Sterculia striata St Hil. Et Nauds seed oil. J. Chromatogr. A 1054(1-2), 235-239 (2004).

91. Barros, A. et al. Mass balance analysis of carbon and nitrogen in industrial scale mixotrophic microalgae cultures. Algal Res. 21, 35-41 (2017).

92. Folch, J., Lees, M. \& Sloane, S. G. A simple method for the isolation and purification of total lipids from animal tissues. J. Biol. Chem. 226, 497-509 (1957).

93. Bonfanti, C. et al. Potential of microalga Isochrysis galbana: Bioactivity and bioaccessibility. Algal. Res. 2018(29), 242-248 (2017).

94. Magalhães, L. M., Segundo, M. A., Reis, S. \& Lima, J. L. F. C. Automatic method for determination of total antioxidant capacity using 2,2-diphenyl-1-picrylhydrazyl assay. Anal. Chim. Acta. 558(1-2), 310-318 (2006).

95. Melo, T. et al. New insights into the anti-inflammatory and antioxidant properties of nitrated phospholipids. Lipids 53(1), $117-131$ (2018)

96. Magalhães, L. M., Barreiros, L., Maia, M. A., Reis, S. \& Segundo, M. A. Rapid assessment of endpoint antioxidant capacity of red wines through microchemical methods using a kinetic matching approach. Talanta 97, 473-483 (2012).

97. Ulbricht T. V., \& Southgate, D. A. T. Coronary heart disease and dietary factors. Lancet 338, 985-992.

98. Lopes, D., Melo, T., Meneses, J., Abreu, M.H., Pereira, R., Domingues, P., Lillebø, A. I., Calado, R., \& Rosário Domingues M. A new look for the red macroalga palmaria palmata: a seafood with polar lipids rich in EPA and with antioxidant properties. Mar. Drugs. 17(9) (2019).

99. Moreira, A. S. P. et al. Seasonal plasticity of the polar lipidome of Ulva rigida cultivated in a sustainable integrated multi-trophic aquaculture. Algal Res. 49, 101958. https://doi.org/10.1016/j.algal.2020.101958 (2020).

100. Monteiro JP, Rey F, Melo T, Moreira ASP, Arbona JF, Skjermo J, Forbord S, Funderud J, Raposo D, Kerrison PD, Perrineau MM, Gachon C, Domingues P, Calado R, Domingues MR. The unique lipidomic signatures of saccharina latissima can be used to pinpoint their geographic origin. Biomolecules. 10(1), 1-17 (2020).

101. da Costa, E., Amaro, H. M., Melo, T., Guedes, A. C., \& Domingues, M.R. Screening for polar lipids, antioxidant, and antiinflammatory activities of Gloeothece sp. lipid extracts pursuing new phytochemicals from cyanobacteria. J. Appl. Phycol. (2020).

102. Pluskal, T., Castillo, S., Villar-Briones, A. \& Orešič, M. MZmine 2: Modular framework for processing, visualizing, and analyzing mass spectrometry-based molecular profile data. BMC Bioinf. 11(395), 1-11 (2010).

\section{Acknowledgements}

The authors would like to acknowledge all staff members of Allmicroalgae Natural Products, S.A. for the kind support in microalgae growth. Thanks are due to the University of Aveiro and FCT / MCT for the financial support to QOPNA ((FCT UID/QUI/00062/2019) and LAQV/REQUIMTE (UIDB/50006/2020), CESAM (UIDB/50017/2020 + UIDP/50017/2020) and to RNEM, Portuguese Mass Spectrometry Network, (LISBOA01-0145-FEDER-402-022125) through national funds and, where applicable, co-financed by the FEDER, within the PT2020 Partnership Agreement. Tiago Alexandre Conde (2020.05678.BD) to FCT for his grant. Daniela Salomé Moderno do Couto (SFRH/BD/138992/2018) is grateful to FCT for her grant. Tânia Melo thanks the research contract under the project Omics 4 Algae: Lipidomic tools for chemical phenotyping, traceability and valorisation of seaweeds from aquaculture as a sustainable source of high added-value compounds (POCI-01-0145-FEDER-030962), funded by Centro2020, through FEDER and PT2020. This is a contribution of Marine Lipidomics laboratory. This work has received funding under the project AlgaValor, from the Portugal 2020 program (Grant agreement nos. POCI-01-0247-FEDER-035234; LISBOA-01-0247-FEDER-035234; ALG-01-0247-FEDER-035234).

\section{Author contributions}

Conceptualization and design: T.A.C, D.C., P.D.; and M.R.D.; methodology and formal analysis: T.A.C, D.C., P.D., M.R.D. and T.M.; validation, T.A.C., D.C., P.D., M.R.D. and T.M.; collection and assembly of data: T.A.C., D.C., P.D., M.R.D. and T.M.; writing-original draft preparation: T.A.C., D.C., P.D., M.R.D. and T.M.; All authors reviewed, read and approved the published version of the manuscript.

\section{Competing interests}

The authors declare no competing interests.

\section{Additional information}

Supplementary Information The online version contains supplementary material available at https://doi. org/10.1038/s41598-021-83455-y.

Correspondence and requests for materials should be addressed to P.D. 
Reprints and permissions information is available at www.nature.com/reprints.

Publisher's note Springer Nature remains neutral with regard to jurisdictional claims in published maps and institutional affiliations.

(c) (i) Open Access This article is licensed under a Creative Commons Attribution 4.0 International License, which permits use, sharing, adaptation, distribution and reproduction in any medium or format, as long as you give appropriate credit to the original author(s) and the source, provide a link to the Creative Commons licence, and indicate if changes were made. The images or other third party material in this article are included in the article's Creative Commons licence, unless indicated otherwise in a credit line to the material. If material is not included in the article's Creative Commons licence and your intended use is not permitted by statutory regulation or exceeds the permitted use, you will need to obtain permission directly from the copyright holder. To view a copy of this licence, visit http://creativecommons.org/licenses/by/4.0/.

(C) The Author(s) 2021 